\title{
First order symmetry operators for the linearized field equation of metric perturbations
}

\author{
Yoji Michishita* \\ Department of Physics, Faculty of Education, Kagoshima University, Kagoshima 890-0065, Japan
}

(Received 7 October 2019; published 20 December 2019)

\begin{abstract}
We determine the general form of the first order linear symmetry operators for the linearized field equation of metric perturbations in the spacetimes of dimension $D \geq 4$. Apart from the part derived easily from the invariance under general coordinate transformations, we find a part consisting of a Killing-Yano 3-form.
\end{abstract}

DOI: $10.1103 /$ PhysRevD.100.124052

\section{INTRODUCTION}

It is often necessary to solve the linearized equations of motion of various fields in given background geometries. Especially the equation of the perturbations of the metric $h_{\mu \nu}$ is important: Its time-dependent solutions indicate how gravitational waves propagate or whether the background is stable. It may also give moduli of the background geometry satisfying the background equation of motion. To make it easier to solve the linearized equation $M_{\lambda \rho}{ }^{\mu \nu} h_{\mu \nu}=0$, where $M_{\lambda \rho}{ }^{\mu \nu}$ is a second order derivative operator defined later in (2.7), we consider symmetry operators of $M_{\lambda \rho}{ }^{\mu \nu}$ i.e., pairs of operators $\left(Q_{\lambda \rho}{ }^{\mu \nu}, S_{\lambda \rho}{ }^{\mu \nu}\right)$ satisfying

$$
Q_{\lambda \rho}{ }^{\kappa \phi} M_{\kappa \phi}{ }^{\mu \nu} h_{\mu \nu}=M_{\lambda \rho}{ }^{\kappa \phi} S_{\kappa \phi}^{\mu \nu} h_{\mu \nu}
$$

Such operators, especially lower order ones, for lower spin fields, or in some classes of backgrounds have been investigated in the literature. Often variants of symmetry operators, such as the ones for the equations of lower spin fields obtained from the original equation by some ansatz for the form of solutions, or the ones connecting solutions to the original equation with solutions to easier equations, have also been considered. The following is a partial list of recent discussion: For spin 0 fields in generalized KerrNUT-(A)dS spacetimes, see [1]. For spin 1/2 fields see e.g., [2-5]. For spin 1 fields under some ansatz in KerrNUT-(A)dS spacetimes see [6,7], and in 4 dimensions see [8]. For spin 3/2 fields see, e.g., [9]. For spin 2 and lower spin fields in Petrov type D spacetimes and higher dimensional extensions, see, e.g., [10-12].

\footnotetext{
"michishita@edu.kagoshima-u.ac.jp
}

Published by the American Physical Society under the terms of the Creative Commons Attribution 4.0 International license. Further distribution of this work must maintain attribution to the author(s) and the published article's title, journal citation, and DOI. Funded by SCOAP ${ }^{3}$.
In this paper, we make no assumption about the form and the signature of the background geometry except that the dimension $D$ is greater than or equal to 4 , and determine the general form of the first order symmetry operators $\left(Q_{\lambda \rho}{ }^{\mu \nu}, S_{\lambda \rho}{ }^{\mu \nu}\right)$ for the equation of $h_{\mu \nu}$ by straightforward calculations. We do not use the background equation of motion at intermediate steps, and at the final step we use it and find the results (3.13) and (3.14). Nontrivial parts of them consist of three parts: a gauge transformation part and a part consisting of a (conformal) Killing vector, which are well known and can be derived easily from the invariance under general coordinate transformations, and a part consisting of a Killin-Yano 3-form. In Sec. II we give conditions for first order symmetry operators, and in Sec. III we show the general solution of the conditions and give conclusions for readers who are interested only in the results. The details of how to solve the conditions is shown in Sec. IV. In the Appendix we briefly summarize properties of conformal Killing vectors and Killing-Yano 3-forms used in the calculations.

\section{PRELIMINARIES}

We consider the metric $g_{\mu \nu}$ in $D$-dimensional spaces, which we decompose into the background metric $G_{\mu \nu}$ and the perturbation $h_{\mu \nu}: g_{\mu \nu}=G_{\mu \nu}+h_{\mu \nu}$. No assumption about the signature of $g_{\mu \nu}$ is made.

Einstein-Hlibert Lagrangian for $g_{\mu \nu}$ (up to the overall constant factor) is given by

$$
\mathcal{L}=\sqrt{|g|}(R(g)-2 \Lambda)
$$

The part linear in $h_{\mu \nu}$ is given by

$$
\begin{aligned}
\left.\mathcal{L}\right|_{h}= & -\sqrt{|G|}\left[R^{\mu \nu}(G)-\frac{1}{2} G^{\mu \nu} R(G)+\Lambda G^{\mu \nu}\right] h_{\mu \nu} \\
& + \text { (total derivative term) }
\end{aligned}
$$


which shows that if there is no matter field the background equation of motion is the following vacuum Einstein equation:

$$
R^{\mu \nu}(G)-\frac{1}{2} G^{\mu \nu} R(G)+\Lambda G^{\mu \nu}=0,
$$

or equivalently,

$$
R^{\mu \nu}(G)=\frac{2}{D-2} \Lambda G^{\mu \nu},
$$

and the following also holds:

$$
R(G)=\frac{2 D}{D-2} \Lambda .
$$

Unless otherwise stated, we do not impose these equations on the background. The part quadratic in $h_{\mu \nu}$ is given by

$\left.\mathcal{L}\right|_{h^{2}}=\sqrt{|G|} h_{\lambda \rho} M^{\lambda \rho \mu \nu} h_{\mu \nu}+($ total derivative term $)$,

where $M^{\lambda \rho \mu \nu}$ is the following Hermitian operator:

$$
\begin{aligned}
M_{\lambda \rho}^{\mu \nu}= & \frac{1}{4}\left(\delta_{\lambda}{ }^{(\mu} \delta_{\rho}^{\nu)} G^{\sigma \tau}-G_{\lambda \rho} G^{\mu \nu} G^{\sigma \tau}+\delta_{\left(\lambda^{\sigma}\right.} \delta_{\rho)}{ }^{\tau} G^{\mu \nu}\right. \\
& \left.+G_{\lambda \rho} G^{(\mu \mid \sigma} G^{\tau \mid \nu)}-2 \delta_{(\lambda}{ }^{\tau} \delta_{\rho)}{ }^{\mu} G^{\nu) \sigma}\right) \nabla_{\sigma} \nabla_{\tau} \\
& +\delta_{(\lambda}{ }^{(\mu} R_{\rho)}{ }^{\nu)}(G)+\frac{1}{8} G_{\lambda \rho} G^{\mu \nu} R(G)-\frac{1}{4} \delta_{\left(\lambda^{\mu}\right.} \delta_{\rho)}{ }^{\nu} R(G) \\
& -\frac{1}{4} G_{\lambda \rho} R^{\mu \nu}(G)-\frac{1}{4} G^{\mu \nu} R_{\lambda \rho}(G) \\
& -\frac{1}{4} \Lambda\left(G_{\lambda \rho} G^{\mu \nu}-2 \delta_{\left(\lambda^{\mu}\right.} \delta_{\rho)}{ }^{\nu}\right),
\end{aligned}
$$

where $\nabla_{\mu}$ is the covariant derivative of the background geometry, and indices are raised and lowered by $G^{\mu \nu}$ and $G_{\mu \nu}$. Henceforth the background Riemann tensor $R_{\lambda \rho \mu \nu}(G)$ is denoted just by $R_{\lambda \rho \mu \nu}$.

The linearized equation of motion for the perturbation is $M_{\lambda \rho}{ }^{\mu \nu} h_{\mu \nu}=0$. If operators $Q_{\lambda \rho}{ }^{\mu \nu}$ and $S_{\lambda \rho}{ }^{\mu \nu}$ satisfying

$$
Q_{\lambda \rho}{ }^{\kappa \phi} M_{\kappa \phi}^{\mu \nu} h_{\mu \nu}=M_{\lambda \rho}{ }^{\kappa \phi} S_{\kappa \phi}^{\mu \nu} h_{\mu \nu}
$$

exist, then we can generate a new solution $S_{\lambda \rho}{ }^{\mu \nu} h_{\mu \nu}$ from a solution $h_{\mu \nu}$. If $S_{\lambda \rho}^{\mu \nu}$ is a commuting operator, i.e., $Q_{\lambda \rho}^{\mu \nu}=S_{\lambda \rho}{ }^{\mu \nu}$, we can use it to classify the solutions by the simultaneous diagonalization with $M_{\lambda \rho}{ }^{\mu \nu}$. Such operators are called symmetry operators. In this paper we determine the general form of symmetry operators which are first order in derivative operator, i.e., from the condition (2.8) for $Q_{\lambda \rho}{ }^{\mu \nu}$ and $S_{\lambda \rho}{ }^{\mu \nu}$ in the following form:

$$
Q_{\lambda \rho}{ }^{\mu \nu}=Q_{\lambda \rho}{ }^{\mu \nu \phi} \nabla_{\phi}+q_{\lambda \rho}{ }^{\mu \nu},
$$

$$
S_{\lambda \rho}^{\mu \nu}=S_{\lambda \rho}^{\mu \nu \phi} \nabla_{\phi}+S_{\lambda \rho}^{\mu \nu},
$$

we determine $Q_{\lambda \rho}{ }^{\mu \nu \phi}, q_{\lambda \rho}{ }^{\mu \nu}, S_{\lambda \rho}{ }^{\mu \nu \phi}$, and $s_{\lambda \rho}{ }^{\mu \nu}$.

Some examples of the symmetry operators can be found easily. The first example is a trivial one: If $Q_{\lambda \rho}{ }^{\mu \nu}$ and $S_{\lambda \rho}{ }^{\mu \nu}$ are proportional to the unit matrix:

$$
S_{\lambda \rho}{ }^{\mu \nu}=Q_{\lambda \rho}{ }^{\mu \nu}=c \delta_{\left(\lambda^{\mu}\right.}{ }^{\mu} \delta_{\rho}{ }^{\nu}, \quad c=\text { const. }
$$

(2.8) is satisfied. To give next examples, we consider infinitesimal general coordinate transformation $x^{\prime \mu}=$ $x^{\mu}-\epsilon^{\mu} \cdot g_{\mu \nu}$ is transformed by this, which we regard as the transformation of the perturbation $h_{\mu \nu}$ :

$$
\delta g_{\mu \nu}=\delta\left(G_{\mu \nu}+h_{\mu \nu}\right)=G_{\mu \nu}+\delta h_{\mu \nu},
$$

and

$$
\delta h_{\mu \nu}=\nabla_{\mu} \epsilon_{\nu}+\nabla_{\nu} \epsilon_{\mu}+\mathcal{L}_{\epsilon} h_{\mu \nu},
$$

where $\mathcal{L}_{\epsilon}$ is the Lie derivative operator along $\epsilon^{\mu}$ :

$$
\begin{aligned}
\mathcal{L}_{\epsilon} h_{\mu \nu} & =\epsilon^{\lambda} \partial_{\lambda} h_{\mu \nu}+\partial_{\mu} \epsilon^{\lambda} h_{\lambda \nu}+\partial_{\nu} \epsilon^{\lambda} h_{\mu \lambda} \\
& =\epsilon^{\lambda} \nabla_{\lambda} h_{\mu \nu}+\nabla_{\mu} \epsilon^{\lambda} h_{\lambda \nu}+\nabla_{\nu} \epsilon^{\lambda} h_{\mu \lambda} .
\end{aligned}
$$

Under this transformation, $\delta \mathcal{L}=\partial_{\mu}\left(\epsilon^{\mu} \mathcal{L}\right)$ for arbitrary $h_{\mu \nu}$, and therefore

$$
\begin{aligned}
0= & \int d^{D} x \delta \mathcal{L} \\
= & \int d^{D} x \sqrt{|G|}\left[-\left(R^{\mu \nu}(G)-\frac{1}{2} G^{\mu \nu} R(G)+\Lambda G^{\mu \nu}\right) \delta h_{\mu \nu}\right. \\
& \left.+\delta h_{\lambda \rho} M^{\lambda \rho \mu \nu} h_{\mu \nu}+h_{\lambda \rho} M^{\lambda \rho \mu \nu} \delta h_{\mu \nu}+O\left(h^{2}, \delta h\right)\right] .
\end{aligned}
$$

If $\epsilon^{\mu}$ is a Killing vector of the background geometry, $\delta h_{\mu \nu}$ contains only terms linear in $h_{\mu \nu}$, and from the terms quadratic in $h_{\mu \nu}$ in the above,

$$
\begin{aligned}
0 & =\int d^{D} x \sqrt{|G|}\left[\left(\mathcal{L}_{\epsilon} h_{\lambda \rho}\right) M^{\lambda \rho \mu \nu} h_{\mu \nu}+h_{\lambda \rho} M^{\lambda \rho \mu \nu} \mathcal{L}_{\epsilon} h_{\mu \nu}\right] \\
& =\int d^{D} x \sqrt{|G|}\left[h_{\lambda \rho}\left(M^{\lambda \rho \mu \nu} \mathcal{L}_{\epsilon}-\mathcal{L}_{\epsilon} M^{\lambda \rho \mu \nu}\right) h_{\mu \nu}\right] .
\end{aligned}
$$

Therefore we expect that $\mathcal{L}_{\epsilon}$ commutes with $M_{\lambda \rho}{ }^{\mu \nu}$ :

$$
\mathcal{L}_{\epsilon} M_{\lambda \rho}{ }^{\mu \nu} h_{\mu \nu}=M_{\lambda \rho}{ }^{\mu \nu} \mathcal{L}_{\epsilon} h_{\mu \nu} .
$$

Indeed this can be directly confirmed by the fact that $\mathcal{L}_{\epsilon}$ commutes with $\nabla_{\mu}$ and $\mathcal{L}_{\epsilon} R_{\rho \mu \nu}^{\lambda}=0$. Note that (2.17) is true even when the background geometry does not satisfy 
the equation of motion. If the background geometry satisfies the vacuum equation of motion, $\mathcal{L}$ has no linear term in $h_{\mu \nu}$, and from the terms in $\delta \mathcal{L}$ linear in $h_{\mu \nu}$,

$$
0=\int d^{D} x \sqrt{|G|}\left[\left(\nabla^{\lambda} \epsilon^{\rho}\right) M_{\lambda \rho}{ }^{\mu \nu} h_{\mu \nu}+h^{\lambda \rho} M_{\lambda \rho}{ }^{\mu \nu} \nabla_{\mu} \epsilon_{\nu}\right]
$$

Since this is true for arbitrary $h_{\mu \nu}$ and $\epsilon^{\mu}$,

$$
M_{\lambda \rho}^{\mu \nu} \nabla_{\mu} \epsilon_{\nu}=0
$$

$$
\epsilon^{\lambda} \nabla^{\rho} M_{\lambda \rho}^{\mu \nu}=0
$$

These give eigenvectors of zero eigenvalue for $M_{\lambda \rho}{ }^{\mu \nu}$ and are regarded as the degrees of freedom of gauge transformation of $h_{\mu \nu}$. We will see these examples appear in the general form of the first order symmetry operators.

Let us write down the condition (2.8) for each order of derivative. Note that indices on covariant derivatives can be symmetrized by the followings:

$$
\begin{gathered}
\nabla_{\sigma} \nabla_{\tau} h_{\mu \nu}=\nabla_{(\sigma} \nabla_{\tau)} h_{\mu \nu}+R_{\sigma \tau(\mu}{ }^{\kappa} h_{\nu) \kappa}, \\
\nabla_{\phi} \nabla_{\sigma} \nabla_{\tau} h_{\mu \nu}=\nabla_{(\phi} \nabla_{\sigma} \nabla_{\tau)} h_{\mu \nu}+R_{\phi(\sigma|\mu|}{ }^{\kappa} \nabla_{\tau)} h_{\kappa \nu}+R_{\phi(\sigma|\nu|}{ }^{\kappa} \nabla_{\tau)} h_{\mu \kappa}+\frac{1}{2} R_{\sigma \tau \mu}{ }^{\kappa} \nabla_{\phi} h_{\kappa \nu}+\frac{1}{2} R_{\sigma \tau \nu}{ }^{\kappa} \nabla_{\phi} h_{\mu \kappa}+\frac{2}{3} R_{\phi(\sigma \tau)}{ }^{\kappa} \nabla_{\kappa} h_{\mu \nu} \\
+\frac{2}{3} \nabla_{(\sigma} R_{\phi) \tau \mu}{ }^{\kappa} h_{\kappa \nu}+\frac{2}{3} \nabla_{(\sigma} R_{\phi) \tau \nu}{ }^{\kappa} h_{\mu \kappa} .
\end{gathered}
$$

Then terms proportional to $\nabla_{(\phi} \nabla_{\sigma} \nabla_{\tau)} h_{\mu \nu}, \nabla_{(\phi} \nabla_{\tau)} h_{\mu \nu}, \nabla_{\phi} h_{\mu \nu}$, and $h_{\mu \nu}$ in (2.8) cancel separately. From the part proportional to $\nabla_{(\phi} \nabla_{\sigma} \nabla_{\tau)} h_{\mu \nu}$,

$$
\begin{aligned}
& Q_{\lambda \rho \mu \nu}{ }^{(\phi} G^{\sigma \tau)}-Q_{\lambda \rho \kappa}{ }^{\kappa(\phi} G^{\sigma \tau)} G_{\mu \nu}+Q_{\lambda \rho}{ }^{(\sigma \tau \phi)} G_{\mu \nu}+Q_{\lambda \rho \kappa}{ }^{\kappa(\phi} \delta_{\mu}{ }^{\sigma} \delta_{\nu}{ }^{\tau)}-2 Q_{\lambda \rho}{ }^{(\tau}{ }_{(\mu}{ }^{\phi} \delta_{\nu)}{ }^{\sigma)} \\
& \quad=S_{\lambda \rho \mu \nu}{ }^{(\phi} G^{\sigma \tau)}-S_{\kappa}{ }^{\kappa} \mu \nu{ }^{(\phi} G^{\sigma \tau)} G_{\lambda \rho}+S_{\kappa}{ }^{\kappa}{ }_{\mu \nu}{ }^{(\phi} \delta_{\lambda}{ }^{\sigma} \delta_{\rho}{ }^{\tau)}+S^{(\sigma \tau}{ }_{\mu \nu}{ }^{\phi)} G_{\lambda \rho}-2 S_{(\lambda}{ }^{(\sigma}{ }_{\mid \mu \nu}{ }^{\phi} \delta_{\rho)}{ }^{\tau)} .
\end{aligned}
$$

From the part proportional to $\nabla_{(\phi} \nabla_{\tau)} h_{\mu \nu}$,

$$
\begin{aligned}
& q_{\lambda \rho}^{\mu \nu} G^{\tau \phi}-q_{\lambda \rho \kappa}{ }^{\kappa} G^{\tau \phi} G^{\mu \nu}+q_{\lambda \rho}{ }^{\tau \phi} G^{\mu \nu}+q_{\lambda \rho \kappa}{ }^{\kappa} G^{\tau(\mu} G^{\nu) \phi}-q_{\lambda \rho}{ }^{\tau(\mu} G^{\nu) \phi}-q_{\lambda \rho}{ }^{\phi(\mu} G^{\nu) \tau} \\
& =s_{\lambda \rho}{ }^{\mu \nu} G^{\tau \phi}-s_{\kappa}{ }^{\mu \mu \nu} G_{\lambda \rho} G^{\tau \phi}+s_{\kappa}{ }^{\kappa \mu \nu} \delta_{(\lambda}{ }^{\tau} \delta_{\rho)}{ }^{\phi}+s^{\tau \phi \mu \nu} G_{\lambda \rho}-2 s_{(\lambda}\left(\tau|\mu \nu| \delta_{\rho)} \phi\right)+2 \nabla^{(\tau} S_{\lambda \rho}{ }^{|\mu \nu| \phi)}-2 \nabla^{(\tau} S_{\kappa}{ }^{|\kappa \mu \nu| \phi)} G_{\lambda \rho} \\
& \left.+2 \nabla_{\sigma} S^{\sigma(\tau|\mu \nu| \phi)} G_{\lambda \rho}+2 \nabla_{(\lambda} S_{|\kappa|}{ }^{\kappa \mu \nu(\tau} \delta_{\rho)}^{\phi)}-2 \nabla_{(\lambda} S_{\rho)}{ }^{(\tau|\mu \nu| \phi)}-2 \nabla_{\kappa} S_{(\lambda}{ }^{{ }^{k \mu \nu}(\tau} \delta_{\rho)} \phi\right) .
\end{aligned}
$$

From the part proportional to $\nabla_{\phi} h_{\mu \nu}$,

$$
\begin{aligned}
& Q_{\lambda \rho}{ }^{(\mu \mid \kappa \sigma} R_{\sigma}{ }^{\phi \mid}{ }_{\kappa}{ }^{\nu)}-\frac{1}{2} Q_{\lambda \rho \kappa}{ }^{\kappa \sigma} R_{\sigma}{ }^{(\mu} G^{\nu) \phi}-\frac{1}{6} Q_{\lambda \rho \kappa}{ }^{\kappa \sigma} R_{\sigma}{ }^{(\mu \nu) \phi}+\frac{1}{2} Q_{\lambda \rho}{ }^{\phi \kappa \sigma} R_{\sigma}{ }^{(\mu \nu)}{ }_{\kappa}+\frac{1}{2} Q_{\lambda \rho}{ }^{\phi(\mu|\kappa|} R_{\kappa}{ }^{\nu)}-\frac{1}{2} Q_{\lambda \rho}{ }^{\sigma \tau \kappa} R_{\kappa \sigma \tau}{ }^{(\mu} G^{\nu) \phi} \\
& +\frac{1}{6} Q_{\lambda \rho}{ }^{\kappa(\mu|\sigma|} R_{\sigma \kappa}{ }^{\nu) \phi}-\frac{1}{2} Q_{\lambda \rho}{ }^{\kappa \sigma \phi} R_{\kappa}{ }^{(\mu \nu)}{ }_{\sigma}+\frac{3}{2} Q_{\lambda \rho}{ }^{\kappa(\mu|\phi|} R_{\kappa}{ }^{\nu)}-\frac{1}{3} Q_{\lambda \rho}{ }^{\mu \nu \kappa} R_{\kappa}{ }^{\phi}+\frac{1}{3} Q_{\lambda \rho \kappa}{ }^{\kappa \sigma} R_{\sigma}{ }^{\phi} G^{\mu \nu}+\frac{1}{3} Q_{\lambda \rho}{ }^{\sigma \tau \kappa} R_{\kappa \sigma \tau}{ }^{\phi} G^{\mu \nu} \\
& -\frac{1}{3} Q_{\lambda \rho}{ }^{\kappa(\mu|\sigma|} R_{\sigma}{ }^{\nu)}{ }_{\kappa}{ }^{\phi}+\frac{1}{4} Q_{\lambda \rho \kappa}{ }^{\kappa \phi} R G^{\mu \nu}-\frac{1}{2} Q_{\lambda \rho}{ }^{\mu \nu \phi} R-\frac{1}{2} Q_{\lambda \rho \kappa}{ }^{\kappa \phi} R^{\mu \nu}-\frac{1}{2} Q_{\lambda \rho}{ }^{\sigma \tau \phi} R_{\sigma \tau} G^{\mu \nu}-\frac{\Lambda}{2}\left(Q_{\lambda \rho \kappa}{ }^{\kappa \phi} G^{\mu \nu}-2 Q_{\lambda \rho}{ }^{\mu \nu \phi}\right) \\
& =\frac{1}{2} \nabla_{\kappa} \nabla^{\kappa} S_{\lambda \rho}{ }^{\mu \nu \phi}-\frac{1}{2} G_{\lambda \rho} \nabla_{\sigma} \nabla^{\sigma} S_{\kappa}{ }^{\kappa \mu \nu \phi}+\frac{1}{2} \nabla_{(\lambda} \nabla_{\rho)} S_{\kappa}{ }^{\kappa \mu \nu \phi}+\frac{1}{2} G_{\lambda \rho} \nabla^{\sigma} \nabla^{\tau} S_{\sigma \tau}{ }^{\mu \nu \phi}-\nabla_{\kappa} \nabla_{(\lambda} S_{\rho)}{ }^{\kappa \mu \nu \phi}+2 S_{(\lambda}{ }^{\kappa \mu \nu \phi} R_{\rho) \kappa}
\end{aligned}
$$

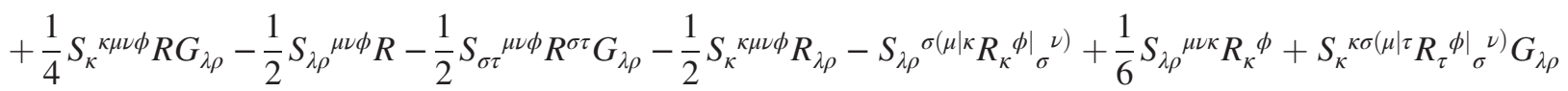

$$
\begin{aligned}
& -\frac{1}{6} S_{\kappa}{ }^{k \mu \nu \sigma} R_{\sigma}{ }^{\phi} G_{\lambda \rho}-S_{\kappa}{ }^{\kappa \sigma(\mu|\tau|} R_{\tau(\lambda|\sigma|}{ }^{\nu)} \delta_{\rho)}{ }^{\phi}-\frac{1}{6} S_{\kappa}{ }^{\kappa \mu \nu \sigma} R_{\sigma(\lambda \rho)}{ }^{\phi}-S^{\kappa \phi \sigma(\mu|\tau|} R_{\tau \kappa \sigma}{ }^{\nu)} G_{\lambda \rho}-\frac{1}{6} S_{\sigma \tau}{ }^{\mu \nu \kappa} R_{\kappa}{ }^{\sigma \tau \phi} G_{\lambda \rho}+S_{(\lambda}{ }^{\kappa \sigma(\mu|\phi|} R_{\rho) \kappa \sigma}{ }^{\nu)}
\end{aligned}
$$

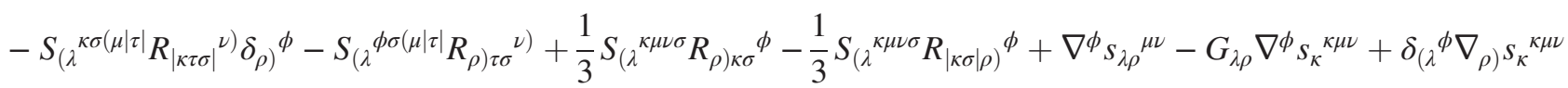

$$
\begin{aligned}
& +G_{\lambda \rho} \nabla_{\kappa} s^{\kappa \phi \mu \nu}-\nabla_{(\lambda} S_{\rho)}{ }^{\phi \mu \nu}-\delta_{(\lambda}{ }^{\phi} \nabla_{|\kappa|} S_{\rho)}{ }^{\kappa \mu \nu}-\frac{\Lambda}{2}\left(S_{\kappa}{ }^{\kappa \mu \nu \phi} G_{\lambda \rho}-2 S_{\lambda \rho}^{\mu \nu \phi}\right) .
\end{aligned}
$$


From the part proportional to $h_{\mu \nu}$,

$$
\begin{aligned}
& \left.\left.\frac{2}{3} Q_{\lambda \rho}{ }^{(\mu|\kappa \sigma|} \nabla_{\sigma} R_{\kappa}{ }^{\nu}\right)+\frac{1}{6} Q_{\lambda \rho}{ }^{(\mu|\kappa \sigma|} \nabla^{\nu)} R_{\kappa \sigma}-\frac{1}{6} Q_{\lambda \rho \kappa}{ }^{\kappa \sigma} \nabla_{\sigma} R^{\mu \nu}-\frac{1}{6} Q_{\lambda \rho \kappa}{ }^{\kappa \sigma} \nabla^{(\mu} R_{\sigma}{ }^{\nu)}-\frac{1}{6} Q_{\lambda \rho}{ }^{\kappa \sigma \tau} \nabla^{(\mu} R_{\tau \kappa \sigma}{ }^{\nu}\right)-\frac{1}{6} Q_{\lambda \rho}{ }^{\kappa \sigma \tau} \nabla_{\tau} R^{(\mu}{ }_{\kappa \sigma}{ }^{\nu)} \\
& \left.+\frac{1}{8} Q_{\lambda \rho \kappa}{ }^{\kappa \sigma} G^{\mu \nu} \nabla_{\sigma} R-\frac{1}{4} Q_{\lambda \rho}{ }^{\mu \nu \kappa} \nabla_{\kappa} R-\frac{1}{4} Q_{\lambda \rho}{ }^{\sigma \tau \kappa} G^{\mu \nu} \nabla_{\kappa} R_{\sigma \tau}-\frac{1}{4} q_{\lambda \rho}{ }^{\kappa \sigma} R^{(\mu}{ }_{\kappa \sigma}{ }^{\nu)}+\frac{3}{4} q_{\lambda \rho}{ }^{\kappa(\mu} R_{\kappa}{ }^{\nu}\right)-\frac{1}{4} q_{\lambda \rho \kappa}{ }^{\kappa} R^{\mu \nu}-\frac{1}{4} q_{\lambda \rho}{ }^{\sigma \tau} G^{\mu \nu} R_{\sigma \tau} \\
& -\frac{1}{4} q_{\lambda \rho}{ }^{\mu \nu} R+\frac{1}{8} q_{\lambda \rho \kappa}{ }^{\kappa} G^{\mu \nu} R-\frac{\Lambda}{4}\left(q_{\lambda \rho \kappa}{ }^{\kappa} G^{\mu \nu}-2 q_{\lambda \rho}{ }^{\mu \nu}\right) \\
& =\frac{1}{3} S_{\lambda \rho}{ }^{\kappa(\mu|\sigma|} \nabla_{\kappa} R^{\nu)}{ }_{\sigma}-\frac{1}{3} S_{\lambda \rho}{ }^{{ }^{(\mu|\sigma|} \mid \nabla^{\nu)}} R_{\kappa \sigma}-\frac{1}{3} G_{\lambda \rho} S_{\kappa}{ }^{\kappa \sigma(\mu|\tau|} \nabla_{\sigma} R^{\nu \nu}{ }_{\tau}+\frac{1}{3} G_{\lambda \rho} S_{\kappa}{ }^{\kappa \sigma(\mu|\tau|} \nabla^{\nu)} R_{\sigma \tau}+\frac{1}{3} G_{\lambda \rho} S^{\sigma \tau \phi \phi(\mu|\kappa|} \nabla_{\sigma} R_{\tau \kappa \phi}{ }^{\nu)} \\
& \left.+\frac{1}{3} S_{\kappa}{ }^{\kappa \sigma(\mu|\tau|} \nabla_{(\lambda} R_{\rho) \tau \sigma}{ }^{\nu)}-\frac{1}{3} S_{(\lambda}{ }^{\kappa \sigma(\mu|\tau|} \nabla_{\rho)} R_{\kappa \tau \sigma}{ }^{\nu)}-\frac{1}{3} S_{(\lambda}{ }^{\kappa \sigma(\mu|\tau|} \nabla_{|\kappa|} R_{\rho) \tau \sigma}{ }^{\nu}\right)+\frac{1}{2} \nabla^{\kappa} S_{\lambda \rho}{ }^{\sigma(\mu|\tau|} R_{\kappa \tau \sigma}{ }^{\nu)}-\frac{1}{2} \nabla^{\sigma} S_{\kappa}{ }^{\kappa \tau(\mu|\phi|} G_{\lambda \rho} R_{\sigma \phi \tau}{ }^{\nu)} \\
& +\frac{1}{2} \nabla_{(\lambda} S_{|\kappa|}{ }^{\kappa \sigma(\mu|\tau|} R_{\rho) \tau \sigma}{ }^{\nu)}+\frac{1}{2} \nabla_{\sigma} S^{\sigma \tau \kappa(\mu|\phi|} G_{\lambda \rho} R_{\tau \phi \kappa}{ }^{\nu)}-\frac{1}{2} \nabla_{(\lambda} S_{\rho)}{ }^{\kappa \sigma(\mu|\tau|} R_{\kappa \tau \sigma}{ }^{\nu)}-\frac{1}{2} \nabla_{\kappa} S_{(\lambda}{ }^{\kappa \sigma(\mu|\tau|} R_{\rho) \tau \sigma}{ }^{\nu)}+\frac{1}{4} \nabla^{\kappa} \nabla_{\kappa} S_{\lambda \rho}{ }^{\mu \nu}
\end{aligned}
$$

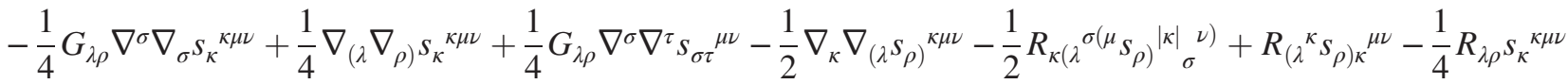

$$
\begin{aligned}
& -\frac{1}{4} G_{\lambda \rho} R^{\sigma \tau} s_{\sigma \tau}{ }^{\mu \nu}-\frac{1}{4} R s_{\lambda \rho}{ }^{\mu \nu}+\frac{1}{8} G_{\lambda \rho} R s_{\kappa}{ }^{\mu \mu \nu}-\frac{\Lambda}{4}\left(s_{\kappa}{ }^{k \mu \nu} G_{\lambda \rho}-2 s_{\lambda \rho}{ }^{\mu \nu}\right) \text {. }
\end{aligned}
$$

In the following sections we solve these conditions. However in 2 dimensions, $R^{\lambda \rho}{ }_{\mu \nu}=R \delta^{[\lambda}{ }_{\mu} \delta^{\rho]}{ }_{\nu}$, and any manifold satisfies vacuum Einstein equation with no cosmological constant. Indeed it can be shown that $M_{\lambda \rho}{ }^{\mu \nu} h_{\mu \nu}=0$ holds for any $h_{\mu \nu}$ if $\Lambda=0$. In 3 dimensions, independent components of Riemann tensor are given in terms of Ricci tensor:

$$
R_{\mu \nu}^{\lambda \rho}=-6 \delta^{[\lambda}{ }_{\mu}^{\rho}{ }_{\nu} \delta^{\sigma]}{ }_{\tau}\left(R^{\tau}{ }_{\sigma}-\frac{1}{2} \delta^{\tau}{ }_{\sigma} R\right),
$$

and this means that any background satisfying vacuum Einstein equation is a space of constant curvature, which has been well studied. Therefore in the following sections we only consider cases of $D \geq 4$.

\section{RESULTS AND CONCLUSIONS}

In this section we summarize our results and give conclusions so that readers who are not interested in the details of the procedure for solving the Eqs. (2.23), (2.24), (2.25), and (2.26) can skip them. The details will be explained in the next section.

First we show the solution to Eq. (2.23) which is purely algebraic:

$$
\begin{aligned}
Q_{\lambda \rho}{ }^{\mu \nu \phi} & =\delta_{\left(\lambda^{\mu}{ }^{\mu} \delta_{\rho)}{ }^{\nu} K^{\phi}+\delta_{(\lambda}{ }^{(\mu} Y_{\rho)}{ }^{\nu) \phi}+G^{\phi(\mu} F_{\lambda \rho}{ }^{\nu)},\right.} \\
S_{\lambda \rho}{ }^{\mu \nu \phi} & =\delta_{\left(\lambda^{\mu}\right.} \delta_{\rho)}{ }^{\nu} K^{\phi}+\delta_{(\lambda}{ }^{(\mu} Y_{\rho)}{ }^{\nu) \phi}+\delta^{\phi}{ }_{(\lambda} H^{\mu \nu}{ }_{\rho)},
\end{aligned}
$$

where $K^{\mu}$ is an arbitrary vector, and $Y_{\mu \nu \lambda}$ is an arbitrary antisymmetric tensor. $F_{\lambda \rho}{ }^{\phi}$ and $H_{\lambda \rho}{ }^{\phi}$ are arbitrary tensors satisfying

$$
F_{\lambda \rho}{ }^{\phi}=F_{\rho \lambda}{ }^{\phi}, \quad H_{\lambda \rho}{ }^{\phi}=H_{\rho \lambda}{ }^{\phi} .
$$

Using the above we obtain the following solution to $(2.24)$ :

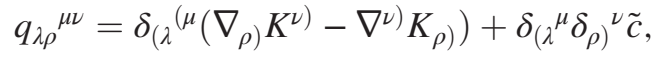

$$
\begin{aligned}
& s_{\lambda \rho}{ }^{\mu \nu}=\delta_{(\lambda}{ }^{(\mu}\left(\nabla_{\rho)} K^{\nu)}-\nabla^{\nu)} K_{\rho)}\right)+\delta_{\left(\lambda^{\mu}\right.} \delta_{\rho)}{ }^{\nu} \tilde{c} \\
& -\frac{2}{D} \delta_{(\lambda}{ }^{\mu} \delta_{\rho)}{ }^{\nu} \nabla_{\kappa} K^{\kappa}+\nabla_{(\lambda} H_{\rho \nu}^{\mu \nu},
\end{aligned}
$$

where $\tilde{c}$ is a scalar function, and it turns out that $K^{\mu}$ must be a conformal Killing vector, and $Y_{\mu \nu \lambda}$ must be a KillingYano tensor.

Then we find that (2.25) is equivalent to the following three relations:

$$
\begin{aligned}
& \partial_{\mu} \tilde{c}=\frac{1}{D-1}\left(R-\frac{2 D}{D-2} \Lambda\right)\left(F_{\mu \kappa}{ }^{\kappa}-\frac{1}{D-2} H_{\kappa}{ }^{\kappa}{ }_{\mu}\right) \\
& +\frac{2}{(D-1)(D-2)}\left(R^{\sigma \tau}-\frac{2}{D-2} \Lambda G^{\sigma \tau}\right) H_{\sigma \tau \mu}, \\
& \partial_{\mu}\left(\nabla_{\kappa} K^{\kappa}\right)=\frac{D}{(D-1)(D+2)}\left(R-\frac{2 D}{D-2} \Lambda\right) \\
& \times\left(F_{\mu \kappa}{ }^{\kappa}+\frac{1}{2} F_{\kappa}{ }^{\kappa}{ }_{\mu}+\frac{D-4}{2(D-2)} H_{\mu \kappa}{ }^{\kappa}\right. \\
& \left.-\frac{1}{D-2} H_{\kappa}{ }^{\kappa}{ }_{\mu}\right)+\frac{2 D}{(D-1)(D-2)(D+2)} \\
& \times\left(R^{\sigma \tau}-\frac{2}{D-2} \Lambda G^{\sigma \tau}\right)\left(H_{\mu \sigma \tau}+H_{\sigma \tau \mu}\right),
\end{aligned}
$$




$$
\begin{aligned}
& \left(R^{\mu \nu}-\frac{1}{2} R G^{\mu \nu}+\Lambda G^{\mu \nu}\right) F_{\lambda \rho}{ }^{\phi}=\frac{1}{2(D-1)(D+2)}\left(R-\frac{2 D}{D-2} \Lambda\right)\left(4 G_{\lambda \rho} G^{\mu \nu} F^{\phi \kappa}{ }_{\kappa}-4 \delta_{(\lambda}{ }^{(\mu} \delta_{\rho)}{ }^{\nu)} F^{\phi \kappa}{ }_{\kappa}-2 D G^{\mu \nu} \delta_{(\lambda}{ }^{\phi} F_{\rho) \kappa}{ }^{\kappa}\right. \\
& -2 D G_{\lambda \rho} G^{\phi(\mu} F^{\nu) \kappa}{ }_{\kappa}+2 D \delta_{(\lambda}{ }^{\phi} \delta_{\rho)}{ }^{(\mu} F^{\nu) \kappa}{ }_{\kappa}+2 D G^{\phi(\mu} \delta_{(\lambda}{ }^{\nu)} F_{\rho) \kappa}{ }^{\kappa}-D G_{\lambda \rho} G^{\mu \nu} F_{\kappa}{ }^{\kappa \phi} \\
& +D \delta_{(\lambda}^{(\mu} \delta_{\rho)}^{\nu)} F_{\kappa}{ }^{\kappa \phi}+2 G^{\mu \nu} \delta_{(\lambda}{ }^{\phi} F^{\kappa}{ }_{|\kappa| \rho)}+2 G_{\lambda \rho} G^{\phi(\mu} F_{\kappa}{ }^{|\kappa| \nu)}-2 \delta_{(\lambda}{ }^{\phi} \delta_{\rho)}{ }^{(\mu} F_{\kappa}{ }^{|\kappa| \nu)} \\
& -2 G^{\phi(\mu} \delta_{(\lambda}{ }^{\nu)} F^{\kappa}{ }_{|\kappa| \rho)}-D G_{\lambda \rho} G^{\mu \nu} H^{\phi \kappa}{ }_{\kappa}+D \delta_{(\lambda}{ }^{(\mu} \delta_{\rho)}{ }^{\nu)} H^{\phi \kappa}{ }_{\kappa}+2 G^{\mu \nu} \delta_{(\lambda}{ }^{\phi} H_{\rho) \kappa}{ }^{\kappa} \\
& \left.+2 G_{\lambda \rho} G^{\phi(\mu} H^{\nu) \kappa}{ }_{\kappa}-2 \delta_{(\lambda}{ }^{\phi} \delta_{\rho)}{ }^{(\mu} H^{\nu) \kappa}{ }_{\kappa}-2 G^{\phi(\mu} \delta_{(\lambda}{ }^{\nu)} H_{\rho) \kappa}{ }^{\kappa}\right) \\
& +\frac{2}{(D-1)(D-2)(D+2)}\left(R^{\sigma \tau}-\frac{1}{2} R G^{\sigma \tau}+\Lambda G^{\sigma \tau}\right) \times\left(-D G_{\lambda \rho} G^{\mu \nu} H_{\sigma \tau}^{\phi}\right. \\
& +D \delta_{(\lambda}{ }^{(\mu} \delta_{\rho)}{ }^{\nu)} H_{\sigma \tau}^{\phi}+2 \delta_{(\lambda}{ }^{\phi} G^{\mu \nu} H_{\rho) \sigma \tau}+2 G_{\lambda \rho} G^{\phi(\mu} H^{\nu)}{ }_{\sigma \tau}-2 \delta_{(\lambda}{ }^{\phi} \delta_{\rho)}{ }^{(\mu} H^{\nu)}{ }_{\sigma \tau} \\
& -2 G^{\phi(\mu} \delta_{(\lambda}{ }^{\nu)} H_{\rho) \sigma \tau}+2 G_{\lambda \rho} G^{\mu \nu} H_{\sigma \tau}{ }^{\phi}-2 \delta_{(\lambda}{ }^{(\mu} \delta_{\rho)}{ }^{\nu)} H_{\sigma \tau}{ }^{\phi}-D \delta_{(\lambda}{ }^{\phi} G^{\mu \nu} H_{|\sigma \tau| \rho)} \\
& \left.-D G_{\lambda \rho} G^{\phi(\mu} H_{\sigma \tau}{ }^{\nu)}+D \delta_{(\lambda}{ }^{\phi} \delta_{\rho)}{ }^{(\mu} H_{\sigma \tau}{ }^{\nu)}+D G^{\phi(\mu} \delta_{(\lambda}{ }^{\nu)} H_{|\sigma \tau| \rho)}\right) \\
& +2\left(R_{(\lambda}{ }^{(\mu}-\frac{1}{2} R \delta_{(\lambda}{ }^{(\mu}+\Lambda \delta_{(\lambda}{ }^{(\mu}\right) H_{\rho) \phi}^{\nu)}-\left(R_{\lambda \rho}-\frac{1}{2} R G_{\lambda \rho}+\Lambda G_{\lambda \rho}\right) H^{\phi(\mu \nu)} \\
& +G_{\lambda \rho}\left(R^{\sigma(\mu}-\frac{2}{D-2} \Lambda G^{\sigma(\mu}\right) Y^{\nu) \phi}{ }_{\sigma}+G^{\mu \nu}\left(R_{(\lambda}^{\sigma}-\frac{2}{D-2} \Lambda \delta^{\sigma}{ }_{(\lambda}\right) Y_{\rho)}{ }_{\sigma}{ }_{\sigma} \\
& -\delta_{(\lambda}{ }^{\phi}\left(R^{\sigma(\mu}-\frac{2}{D-2} \Lambda G^{\sigma(\mu}\right) Y^{\nu)}{ }_{\rho) \sigma}+G^{\phi(\mu}\left(R^{|\sigma|}{ }_{(\lambda}-\frac{2}{D-2} \Lambda G^{|\sigma|}{ }_{(\lambda}\right) Y^{\nu)}{ }_{\rho) \sigma} \\
& +\delta_{(\lambda}\left(\mu\left(R^{\nu) \sigma}-\frac{2}{D-2} \Lambda G^{\nu) \sigma}\right) Y_{\rho) \sigma}^{\phi}-\delta_{(\lambda}{ }^{(\mu}\left(R_{\rho)}{ }^{|\sigma|}-\frac{2}{D-2} \Lambda G_{\rho)}{ }^{|\sigma|}\right) Y^{\nu) \phi} .\right.
\end{aligned}
$$

Note that in these equations Riemann tensor (with no indices contracted) does not appear, and Ricci tensor and $\Lambda$ appear only in the form of the background equations of motion (2.3), (2.4), or (2.5). The last equation (3.8) is somewhat complicated and we obtain no simpler relation from it. However, as is clear from its expression, it is trivially satisfied if (2.3) or (2.4) is satisfied, and (3.6) and (3.7) mean that $\tilde{c}$ and $\nabla_{\kappa} K^{\kappa}$ are constants.

Finally we find that (2.26) is equivalent to the following three relations:

$$
\begin{aligned}
& 0=\frac{(D-2)}{8 D^{2}(D-1)}\left(G_{\lambda \rho} G^{\mu \nu}-D \delta_{\left(\lambda^{\mu}\right.} \delta_{\rho)}^{\nu}\right) \nabla_{\kappa}\left[\left(R-\frac{2 D}{D-2} \Lambda\right) F_{\sigma}^{\kappa \sigma}\right]+\frac{(D-4)}{4(D-2)}\left(R-\frac{2 D}{D-2} \Lambda\right) \delta_{(\lambda}{ }^{\mu} \nabla_{|\kappa|} H_{\rho)}{ }^{\nu) \kappa} \\
& +\frac{1}{4 D(D-2)}\left(R-\frac{2 D}{D-2} \Lambda\right)\left(D G_{\lambda \rho} \nabla_{\kappa} H^{\mu \nu \kappa}-(D-4) G^{\mu \nu} \nabla_{\kappa} H_{\lambda \rho}{ }^{\kappa}\right) \\
& -\frac{1}{8 D(D-1)(D-2)}\left(R-\frac{2 D}{D-2} \Lambda\right)\left[(D+2) G_{\lambda \rho} G^{\mu \nu}+D(D-4) \delta_{\left(\lambda^{\mu}\right.} \delta_{\rho)}{ }^{\nu}\right] \nabla_{\kappa} H_{\sigma}{ }^{\sigma \kappa} \\
& +\frac{1}{4 D^{2}(D-1)}\left(R-\frac{2 D}{D-2} \Lambda\right)\left(D \delta_{\left(\lambda^{\mu}\right.} \delta_{\rho)}^{\nu}-G_{\lambda \rho} G^{\mu \nu}\right) \nabla_{\kappa} H^{\kappa \sigma}{ }_{\sigma}
\end{aligned}
$$

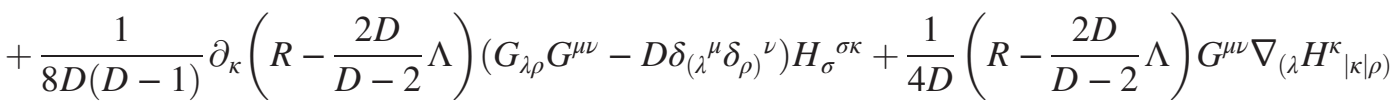

$$
\begin{aligned}
& +\frac{1}{4 D} \partial_{\kappa}\left(R-\frac{2 D}{D-2} \Lambda\right)\left(D \delta_{(\lambda}{ }^{(\mu} H_{\rho)}{ }^{\nu) \kappa}-G^{\mu \nu} H_{\lambda \rho}{ }^{\kappa}\right)-\frac{1}{4}\left(R-\frac{2 D}{D-2} \Lambda\right) \nabla_{(\lambda} H_{\rho)}^{\mu \nu} \\
& +\frac{1}{2 D^{2}(D-1)}\left(R_{\kappa \sigma}-\frac{2}{D-2} \Lambda G_{\kappa \sigma}\right)\left(G_{\lambda \rho} G^{\mu \nu}-D \delta_{(\lambda}{ }^{\mu} \delta_{\rho)}{ }^{\nu}\right) \nabla^{\kappa} H^{\sigma \tau}{ }_{\tau} \\
& +\frac{1}{2(D-1)(D-2)}\left(R_{\kappa \sigma}-\frac{2}{D-2} \Lambda G_{\kappa \sigma}\right)\left(G_{\lambda \rho} G^{\mu \nu}-\delta_{\left(\lambda^{\mu}\right.} \delta_{\rho)}{ }^{\nu}\right) \nabla^{\kappa} H_{\tau}^{\tau \sigma}+\frac{1}{D-2}\left(R_{\kappa \sigma}-\frac{2}{D-2} \Lambda G_{\kappa \sigma}\right) \delta_{(\lambda}{ }^{(\mu} \nabla^{|\kappa|} H_{\rho)}{ }^{\nu) \sigma}
\end{aligned}
$$




$$
\begin{aligned}
& -\frac{1}{2 D(D-2)}\left(R_{\kappa \sigma}-\frac{2}{D-2} \Lambda G_{\kappa \sigma}\right)\left(D G_{\lambda \rho} \nabla^{\kappa} H^{\mu \nu \sigma}+2 G^{\mu \nu} \nabla^{\kappa} H_{\lambda \rho}{ }^{\sigma}\right)-\frac{1}{2 D}\left(R_{\kappa(\lambda}-\frac{2}{D-2} \Lambda G_{\kappa(\lambda}\right) G^{\mu \nu} \nabla^{\kappa} H^{\sigma}{ }_{|\sigma| \rho)} \\
& +\frac{1}{2}\left(R_{\kappa(\lambda}-\frac{2}{D-2} \Lambda G_{\kappa(\lambda}\right) \nabla^{\kappa} H_{\rho \nu}^{\mu \nu}+\frac{1}{4 D} \nabla_{\kappa}\left[\left(R_{\lambda \rho}-\frac{2}{D-2} \Lambda G_{\lambda \rho}\right) G^{\mu \nu} H_{\sigma}{ }^{\sigma \kappa}\right]-\frac{1}{4} \nabla_{\kappa}\left[\left(R_{\lambda \rho}-\frac{2}{D-2} \Lambda G_{\lambda \rho}\right) H^{\mu \nu \kappa}\right]
\end{aligned}
$$

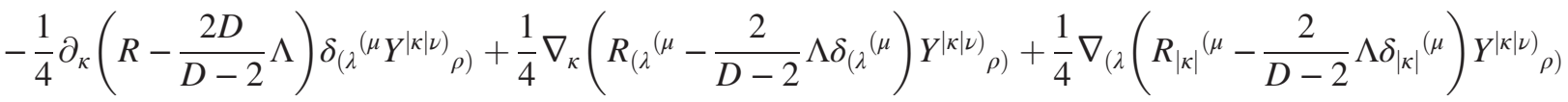

$$
\begin{aligned}
& -\frac{1}{4} \nabla^{(\mu}\left(R_{\kappa(\lambda}-\frac{2}{D-2} \Lambda G_{\kappa(\lambda}\right) Y^{|\kappa| \nu)}{ }_{\rho)}-\frac{1}{4} \nabla_{\kappa}\left(R_{\sigma(\lambda}-\frac{2}{D-2} \Lambda G_{\sigma(\lambda)}\right) \delta_{\rho)}^{(\mu} Y^{|\kappa \sigma| \nu)} \\
& +\frac{1}{4} \nabla_{\kappa}\left(R_{\sigma}{ }^{(\mu}-\frac{2}{D-2} \Lambda \delta_{\sigma}{ }^{(\mu}\right) \delta_{(\lambda}{ }^{\nu)} Y_{\rho)}^{\kappa \sigma}-\frac{1}{2 D} \nabla_{\kappa}\left(R_{\sigma(\lambda}-\frac{2}{D-2} \Lambda G_{\sigma(\lambda}\right) G^{\mu \nu} Y_{\rho)}^{\kappa \sigma},
\end{aligned}
$$

$$
\begin{aligned}
2\left(R^{\kappa \sigma}-\frac{2}{D-2} \Lambda G^{\kappa \sigma}\right) \nabla^{(\mu} H_{\kappa \sigma}{ }^{\nu)}= & -(D-2) \nabla^{(\mu}\left[\left(R-\frac{2 D}{D-2} \Lambda\right) F^{\nu) \kappa}{ }_{\kappa}\right]+\frac{(D-2)^{2}}{2 D^{2}} G^{\mu \nu} \nabla_{\kappa}\left[\left(R-\frac{2 D}{D-2} \Lambda\right) F_{\sigma}^{\kappa \sigma}\right] \\
& +\frac{(D-1)(D-2)^{2}}{2 D} \nabla_{\kappa}\left(R-\frac{2 D}{D-2} \Lambda\right) H^{\mu \nu \kappa}+\nabla^{(\mu}\left(R-\frac{2 D}{D-2} \Lambda\right) H_{\kappa}{ }^{|\kappa| \nu)} \\
& -\frac{D^{2}-2 D+2}{2 D} G^{\mu \nu} \nabla_{\kappa}\left(R-\frac{2 D}{D-2} \Lambda\right) H_{\sigma}{ }^{\sigma \kappa} \\
& +\left(R-\frac{2 D}{D-2} \Lambda\right)\left[\frac{(D-1)(D-2)(D-4)}{2 D} \nabla^{\kappa} H^{\mu \nu}{ }_{\kappa}\right. \\
& \left.+\frac{2 D^{2}-3 D+2}{D} \nabla^{(\mu} H_{\kappa}{ }^{|\kappa| \nu)}-\frac{D^{2}+2}{2 D} G^{\mu \nu} \nabla^{\kappa} H^{\sigma}{ }_{\sigma \kappa}-\frac{D-2}{D^{2}} G^{\mu \nu} \nabla_{\kappa} H^{\kappa \sigma}{ }_{\sigma}\right] \\
& +\frac{2(D-1)^{2}}{D} \nabla_{\kappa}\left[\left(R^{\mu \nu}-\frac{2}{D-2} \Lambda G^{\mu \nu}\right) H_{\sigma}{ }^{\sigma \kappa}\right]-2 \nabla^{(\mu}\left(R_{\kappa \sigma}-\frac{2}{D-2} \Lambda G_{\kappa \sigma}\right) H^{|\kappa \sigma| \nu)} \\
& -\frac{4(D-1)^{2}}{D}\left(R_{\kappa}{ }^{(\mu}-\frac{2}{D-2} \Lambda \delta_{\kappa}{ }^{(\mu}\right) \nabla^{\mid \kappa} H_{\sigma}{ }^{\sigma \mid \nu)} \\
& +2\left(R_{\kappa \sigma}-\frac{2}{D-2} \Lambda G_{\kappa \sigma}\right)\left[G^{\mu \nu} \nabla^{\kappa} H_{\tau}{ }_{\tau}^{\tau \sigma}+\frac{D_{-2}}{D^{2}} G^{\mu \nu} \nabla^{\kappa} H^{\sigma \tau}{ }_{\tau}+\frac{(D-1)(D-2)}{D} \nabla^{\kappa} H^{\mu \nu \sigma}\right] \\
& +\frac{(D-1)\left(D^{2}-6 D+4\right)}{D} \nabla_{\kappa}\left(R_{\sigma}{ }^{(\mu}-\frac{2}{D-2} \Lambda \delta_{\sigma}{ }^{(\mu}\right) Y^{|\kappa \sigma| \nu)},
\end{aligned}
$$

$$
\begin{aligned}
2\left(R^{\kappa \sigma}-\frac{2}{D-2} \Lambda G^{\kappa \sigma}\right) \nabla^{(\mu} H^{\nu \nu}{ }_{\kappa \sigma}= & \frac{2(D-2)}{D^{2}} G^{\mu \nu} \nabla_{\kappa}\left[\left(R-\frac{2 D}{D-2} \Lambda\right) F^{\kappa \sigma}{ }_{\sigma}\right]-\frac{D-2}{2} \nabla^{(\mu}\left[\left(R-\frac{2 D}{D-2} \Lambda\right) F_{\kappa}{ }_{\kappa}^{|\kappa| \nu)}\right] \\
& -\frac{D-4}{2} \nabla^{(\mu}\left[\left(R-\frac{2 D}{D-2} \Lambda\right) H^{\nu)_{\kappa}}{ }_{\kappa}\right]+\left(R-\frac{2 D}{D-2} \Lambda\right)\left[-\frac{(D-1)(D-4)}{D} \nabla^{(\mu} H_{\kappa}{ }^{|\kappa| \nu)}\right. \\
& \left.+\frac{2(D-1)(D-4)}{D} \nabla_{\kappa} H^{\mu \nu \kappa}-\frac{D-4}{2 D} G^{\mu \nu} \nabla_{\kappa} H_{\sigma}{ }^{\sigma \kappa}-\frac{4}{D^{2}} G^{\mu \nu} \nabla_{\kappa} H^{\kappa \sigma}{ }_{\sigma}\right] \\
& +\frac{2(D-1)(D-2)}{D} \nabla_{\kappa}\left(R-\frac{2 D}{D-2} \Lambda\right) H^{\mu \nu \kappa}-\frac{3 D-4}{2 D} G^{\mu \nu} \nabla_{\kappa}\left(R-\frac{2 D}{D-2} \Lambda\right) H_{\sigma}{ }^{\sigma \kappa} \\
& -\frac{(D-1)(D-4)}{D} \nabla_{\kappa}\left[\left(R^{\mu \nu}-\frac{2}{D-2} \Lambda G^{\mu \nu}\right) H_{\sigma}{ }^{\sigma \kappa}\right]
\end{aligned}
$$




$$
\begin{aligned}
& +\frac{2(D-1)(D-4)}{D}\left(R_{\kappa}{ }^{(\mu}-\frac{2}{D-2} \Lambda \delta_{\kappa}{ }^{(\mu}\right) \nabla^{\mid \kappa} H_{\sigma}{ }^{\sigma \mid \nu)} \\
& +\left(R_{\kappa \sigma}-\frac{2}{D-2} \Lambda G_{\kappa \sigma}\right)\left[\frac{8}{D^{2}} G^{\mu \nu} \nabla^{\kappa} H_{\tau}^{\sigma \tau}-2 G^{\mu \nu} \nabla^{\kappa} H_{\tau}{ }^{\tau \sigma}+\frac{8(D-1)}{D} \nabla^{\kappa} H^{\mu \nu \sigma}\right] \\
& -2 \nabla^{(\mu}\left(R_{\kappa \sigma}-\frac{2}{D-2} \Lambda G_{\kappa \sigma}\right) H^{\nu) \kappa \sigma} \\
& -\frac{(D-1)\left(D^{2}-4 D+8\right)}{D} \nabla_{\kappa}\left(R_{\sigma}{ }^{(\mu}-\frac{2}{D-2} \Lambda \delta_{\sigma}{ }^{(\mu)}\right) Y^{|\kappa \sigma| \nu)}-\frac{2(D+2)}{D} \Lambda G^{\mu \nu} \nabla_{\kappa} K^{\kappa} .
\end{aligned}
$$

These also look complicated, but again the Ricci tensor and $\Lambda$ appear only in the combination of the background equations of motion, except in the last term of the last equation (3.11). So if the background equation of motion is satisfied, they are drastically simplified: (3.9) and (3.10) are trivially satisfied, and (3.11) reduces to

$$
\Lambda \nabla_{\kappa} K^{\kappa}=0
$$

In fact this gives no further restriction on $K^{\mu}$ because this is satisfied by any conformal Killing vector with the condition $\nabla_{\kappa} K^{\kappa}=$ const. in the backgrounds satisfying the vacuum equation of motion. [See (4.73).] With (3.12) the final condition for $K^{\mu}$ slightly varies with the value of $\Lambda$, and if the background equation of motion is satisfied, the final form of the symmetry operators are given as follows:

$$
\begin{aligned}
Q_{\lambda \rho}{ }^{\mu \nu} h_{\mu \nu}= & \mathcal{L}_{K} h_{\lambda \rho}+Y_{(\lambda}{ }^{\kappa \phi} \nabla_{|\phi|} h_{\rho) \kappa}+\left(c+\frac{2}{D} \nabla_{\kappa} K^{\kappa}\right) h_{\lambda \rho} \\
& +F_{\lambda \rho}{ }^{\mu} \nabla^{\nu} h_{\mu \nu}, \\
S_{\lambda \rho}{ }^{\mu \nu} h_{\mu \nu}= & \mathcal{L}_{K} h_{\lambda \rho}+Y_{\left(\lambda^{\kappa \phi}\right.} \nabla_{|\phi|} h_{\rho) \kappa}+c h_{\lambda \rho}+\nabla_{(\lambda}\left(H^{\mu \nu}{ }_{\rho)} h_{\mu \nu}\right),
\end{aligned}
$$

where $c=\tilde{c}-\frac{4}{D} \nabla_{\kappa} K^{\kappa}$ is a constant, $Y_{\mu \nu \lambda}$ is a Killing-Yano 3-form, $H_{\lambda}^{\mu \nu}=H_{\lambda}^{\nu \mu}$ is an arbitrary tensor, $F_{\lambda \rho}{ }^{\mu}=F_{\rho \lambda}{ }^{\mu}$ is an arbitrary tensor, and $K^{\mu}$ is a conformal Killing vector satisfying the condition $\nabla_{\kappa} K^{\kappa}=$ const. if $\Lambda=0$, or $K^{\mu}$ is a Killing vector if $\Lambda \neq 0$.

The above result is obtained for $D \geq 4$, but it can be confirmed that it gives a solution even for $D=3$, although it may not be general solution.

The terms proportional to $c$ in (3.13) and (3.14) correspond to (2.11). The term consisting of $H^{\mu \nu}{ }_{\lambda}$ in (3.14) corresponds to (2.19), and the term consisting of $F_{\lambda \rho}{ }^{\mu}$ in (3.13) corresponds to (2.20). The first terms in the right-hand side of (3.13) and (3.14) correspond to (2.17). These are anticipated from the invariance under general coordinate transformations, but the terms proportional to $Y_{\mu \nu \lambda}$ are somewhat unexpected, and give a commuting operator. Some of the eigenfunctions of this term may give nontrivial deformations of the background geometry, and correspond to the moduli of Einstein manifolds. Odd dimensional KerrNUT-(A)dS spacetime (See, e.g., [13] and references therein.) is an example of background manifolds admitting Killing-Yano 3-forms. Sasakian manifolds, 6D nearly Kähler manifolds, 7D weak $G_{2}$ manifolds, and the sphere $S^{D}$ of scalar curvature $D(D-1)$ are Euclidean examples [14]. In the case of $\Lambda=0$, nonzero constant $\nabla_{\kappa} K^{\kappa}$ gives a noncommuting symmetry operator, and it is also interpreted as a result of general coordinate transformation, because if $h_{\mu \nu}$ is a solution, $\nabla_{\mu} K_{\nu}+\nabla_{\nu} K_{\mu}+\mathcal{L}_{K} h_{\mu \nu}$ is also a solution, which is in the form of (2.13). In fact, the conformal transformation with $\nabla_{\kappa} K^{\kappa}=$ const. is a constant rescaling, and the difference between $S_{\lambda \rho}{ }^{\mu \nu}$ and $Q_{\lambda \rho}{ }^{\mu \nu}$ comes from the rescaling of the background metric in $M_{\lambda \rho}{ }^{\mu \nu}$.

By analyses similar to ours given in the next section we can construct general form of higher order symmetry operators in principle. However such calculations become increasingly difficult as the order of the operators becomes higher and higher. One of immediate methods to give higher order operators is to take products of first order operators. Although it gives no more information on the solutions than the first order operators, it may give a hint about the general forms of higher operators.

\section{A PROCEDURE FOR SOLVING THE CONDITIONS FOR THE SYMMETRY OPERATORS}

In this section we show the details of the procedure for solving the conditions (2.23), (2.24), (2.25), and (2.26). Since full expressions of equations appearing at intermediate steps are often lengthy, we only show the algorithm for solving them and do not show unnecessary details. Our basic strategy is to express tensors in terms of tensors with fewer free indices.

\section{A. Analysis of (2.23) for $S^{\lambda \rho}{ }_{\mu \nu}{ }^{\phi}$}

First let us solve (2.23), which is purely algebraic and does not contain derivatives. By contracting $\sigma$ and $\tau$ in $(2.23)$, we obtain 


$$
\begin{aligned}
& \left.D\left(S^{\lambda \rho}{ }_{\mu \nu}{ }^{\phi}-S_{\kappa}{ }^{\kappa} \mu \nu{ }^{\phi} G^{\lambda \rho}\right)-2 S^{(\lambda|\phi|}{ }_{\mu \nu} \rho\right)+2 S_{\kappa}{ }^{\kappa}{ }_{\mu \nu}{ }^{(\lambda} G^{\rho) \phi} \\
& +2 S^{\phi}{ }_{\kappa \mu \nu}{ }^{\kappa} G^{\lambda \rho}-2 S^{(\lambda \mu \nu}{ }_{\kappa \mu \nu}{ }^{|\kappa|} G^{\rho) \phi} \\
& =\left(\text { terms proportional to } Q_{\gamma \delta}^{\alpha \beta \epsilon}\right) \text {. }
\end{aligned}
$$

By symmetrizing the indices $\lambda, \rho$ and $\phi$ in this equation, we obtain

$$
\begin{aligned}
& \left.(D-2)\left(S_{\mu \nu}^{(\lambda \rho}{ }_{\mu \nu}\right)-S_{\kappa}{ }^{\kappa}{ }_{\mu \nu}{ }^{(\phi} G^{\lambda \rho)}\right) \\
& =\left(\text { terms proportional to } Q_{\gamma \delta}{ }^{\alpha \beta \epsilon}\right) \text {. }
\end{aligned}
$$

With this equation $S^{(\lambda \rho}{ }_{\mu \nu}{ }^{\phi)}$ can be expressed by $S_{K}{ }^{\kappa}{ }_{\mu \nu}\left(\phi G^{\lambda \rho)}\right.$ and terms proportional to $Q_{\gamma \delta}{ }^{\alpha \beta \epsilon}$. Then using it and

$$
\left.S_{\mu \nu}^{(\lambda|\phi|}{ }_{\mu \nu}^{\rho)}=-\frac{1}{2} S_{\mu \nu}^{\lambda \rho}{ }^{\phi}+\frac{3}{2} S^{(\lambda \rho}{ }_{\mu \nu} \phi\right),
$$

$\left.S^{(\lambda|\phi|}{ }_{\mu \nu} \rho\right)$ can be expressed by $S^{\lambda \rho}{ }_{\mu \nu}{ }^{\phi}, S_{\kappa}{ }^{\kappa}{ }_{\mu \nu}{ }^{(\phi} G^{\lambda \rho)}$ and terms proportional to $Q_{\gamma \delta}^{\alpha \beta \epsilon}$. By using it we can eliminate $\left.S_{\mu \nu}^{(\lambda|\phi|} \rho\right)$ in (4.1), and we obtain

$$
\begin{aligned}
S_{\mu \nu}^{\lambda \rho}{ }^{\phi}= & S_{\kappa}{ }^{\kappa}{ }_{\mu \nu}{ }^{\phi} G^{\lambda \rho} \\
& +\frac{1}{(D+1)}\left[-2 S^{\phi}{ }_{\kappa \mu \nu}{ }^{\kappa} G^{\lambda \rho}+2 S^{(\lambda \mu \nu}{ }{ }^{|\kappa|} G^{\rho) \phi}\right] \\
& +\left(\text { terms proportional to } Q_{\gamma \delta}{ }^{\alpha \beta \epsilon}\right) .
\end{aligned}
$$

By contracting $\lambda$ and $\rho$ in (4.4),
$S_{\kappa}{ }^{\kappa}{ }_{\mu \nu}{ }^{\phi}=\frac{2}{D+1} S^{\phi}{ }_{\kappa \mu \nu}{ }^{\kappa}+\left(\right.$ terms proportional to $\left.Q_{\gamma \delta}{ }^{\alpha \beta \epsilon}\right)$,

and the contraction $\rho=\phi$ in (4.4) leads to an equation of similar (but different) form:

$S_{\kappa}{ }^{\kappa}{ }_{\mu \nu}{ }^{\phi}=\frac{2}{D+1} S^{\phi}{ }_{\kappa \mu \nu}{ }^{\kappa}+\left(\right.$ terms proportional to $\left.Q_{\gamma \delta}{ }^{\alpha \beta \epsilon}\right)$.

The difference of (4.5) and (4.6) gives a relation containing only $Q_{\gamma \delta}{ }^{\alpha \beta \epsilon}$. Using it we can simplify (4.5):

$$
\begin{aligned}
& S_{\kappa}{ }^{\kappa}{ }_{\mu \nu}{ }^{\phi}=\frac{2}{D+1} S^{\phi}{ }_{\kappa \mu \nu}{ }^{\kappa} \\
& -\frac{1}{(D-2)(D+1)}\left[D Q_{\kappa}{ }^{\kappa}{ }_{\mu \nu}{ }^{\phi}-2 Q_{\kappa}{ }^{\kappa \phi}{ }_{(\mu \nu)}\right. \\
& -D Q_{\kappa}{ }^{\kappa}{ }^{\sigma \phi}{ }^{\sigma} G_{\mu \nu}+2 Q_{\kappa}{ }^{\kappa \phi}{ }_{\sigma}{ }^{\sigma} G_{\mu \nu}+2 Q_{\kappa}{ }^{\kappa} \sigma{ }^{\sigma}{ }_{(\mu} \delta_{\nu}{ }^{\phi} \\
& -2 Q_{\kappa}{ }^{\kappa} \sigma\left(\mu{ }^{\sigma} \delta_{\nu}{ }^{\phi}\right] \text {. }
\end{aligned}
$$

By using this (4.4) can be simplified further:

$$
\begin{aligned}
S_{\mu \nu}^{\lambda \rho}{ }_{\mu \nu}= & \frac{2}{D+1} S_{\kappa \mu \nu}^{(\lambda}|\kappa| G^{\rho) \phi} \\
& +\left(\text { terms proportional to } Q_{\gamma \delta}{ }^{\alpha \beta \epsilon}\right) .
\end{aligned}
$$

Each term in the right-hand side of (2.23) can be rewritten by this, and we obtain

$$
\begin{aligned}
& 0=\frac{1}{(D-2)(D+1)}\left[2 Q_{\lambda \rho \mu \nu}{ }^{(\phi} G^{\sigma \tau)}+2(D-2)(D+1) Q_{\lambda \rho(\mu}{ }^{(\tau \phi} \delta_{\nu)}{ }^{\sigma)}-(D-2)(D+1) Q_{\lambda \rho}{ }^{(\sigma \tau \phi)} G_{\mu \nu}\right. \\
& -2(D-1) Q_{\lambda \rho}{ }_{(\phi \nu)} G^{\sigma \tau)}+2 D Q^{(\phi}{ }_{(\lambda|\mu \nu| \rho)} G^{\sigma \tau)}-4 Q^{(\phi}{ }_{(\lambda \rho)(\mu \nu)} G^{\sigma \tau)}+D(D+1) Q_{\mu \nu}^{(\sigma \tau}{ }_{\mu \nu} G_{\lambda \rho}-2(D+1) Q^{(\sigma \tau \phi)}{ }_{(\mu \nu)} G_{\lambda \rho}
\end{aligned}
$$

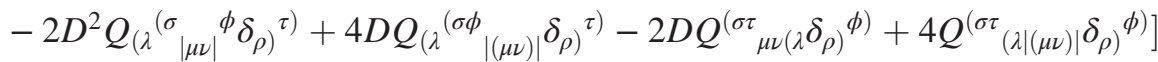

$$
\begin{aligned}
& + \text { (terms proportional to } Q_{\gamma \delta}^{\alpha \beta \epsilon} \text { with some pairs of indices contracted). }
\end{aligned}
$$

Note that this no longer contains $S^{\gamma \delta}{ }_{\alpha \beta}{ }^{\epsilon}$. Therefore (4.8) exhaustively contains information on $S^{\gamma \delta}{ }_{\alpha \beta}{ }^{\epsilon}$.

\section{B. Analysis of (2.23) for $Q^{\lambda \rho}{ }_{\mu \nu}{ }^{\phi}$}

By contracting $\mu$ and $\nu$ in (4.9), we obtain

$$
Q_{\lambda \rho}{ }^{(\phi \sigma \tau)}=\left(\text { terms proportional to } Q_{\gamma \delta}{ }^{\alpha \beta \epsilon}\right. \text { with some pairs of indices contracted), }
$$

and contracting $\lambda$ and $\rho$ in (4.9) we obtain

$$
\begin{aligned}
& 0=D Q_{\mu \nu}^{(\sigma \tau}{ }^{\phi)}-2 Q^{(\sigma \tau \phi)}(\mu \nu) \\
& + \text { (terms proportional to } Q_{\gamma \delta}{ }^{\alpha \beta \epsilon} \text { with some pairs of indices contracted) } \\
& \left.=(D+1) Q_{\mu \nu}^{(\sigma \tau} \phi\right) \\
& -\left(Q_{(\mu \nu)}^{(\sigma \tau \phi)}+Q_{\left(\mu{ }_{\nu)}\right.}^{(\sigma \tau}+Q_{\mu \nu}^{(\sigma \tau}{ }_{\mu \nu}^{\phi)}\right) \\
& + \text { (terms proportional to } Q_{\gamma \delta}^{\alpha \beta \epsilon} \text { with some pairs of indices contracted). }
\end{aligned}
$$


The second line of the last expression of the above equation is symmetric under the interchange of the last three indices, and therefore it can be eliminated by (4.10). Then

$$
Q_{\mu \nu}^{(\sigma \tau}{ }_{\mu \nu}^{\phi)}=\text { (terms proportional to } Q_{\gamma \delta}^{\alpha \beta \epsilon} \text { with some pairs of indices contracted). }
$$

From (4.10) and (4.12),

$$
\begin{aligned}
Q^{\sigma \tau \phi(\mu \nu)} & =Q^{\phi(\sigma|\mu \nu| \tau)}+\frac{3}{2}\left(Q^{\sigma \tau(\mu \nu \phi)}-Q^{(\sigma \tau|\mu \nu| \phi)}\right) \\
& =Q^{\phi(\sigma|\mu \nu| \tau)}+\text { (terms proportional to } Q_{\gamma \delta}{ }^{\alpha \beta \epsilon} \text { with some pairs of indices contracted). }
\end{aligned}
$$

Rewriting the third term of (4.9) by (4.10), the seventh term by (4.12), the fourth, sixth, eighth, tenth, and twelfth terms by (4.13), we obtain

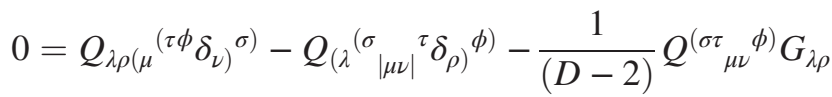

$$
\begin{aligned}
& + \text { (terms proportional to } Q_{\gamma \delta}{ }^{\alpha \beta \epsilon} \text { with some pairs of indices contracted). }
\end{aligned}
$$

The third term of the above can be rewritten by (4.12):

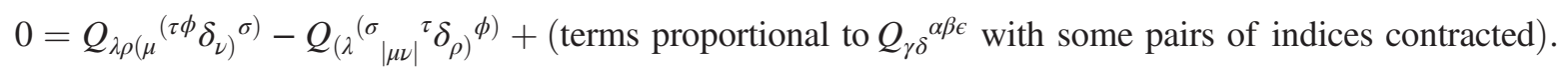

Contracting $\nu$ and $\phi$ in the above,

$$
0=(D+3) Q_{\lambda \rho \mu}{ }^{(\sigma \tau)}-2 Q_{(\lambda}{ }^{(\sigma}{ }_{|\mu| \rho)}^{\tau)}+\left(\text { terms proportional to } Q_{\gamma \delta}{ }^{\alpha \beta \varepsilon}\right. \text { with some pairs of indices contracted). }
$$

From (4.10)

$$
\begin{aligned}
Q_{\lambda \rho}{ }^{\mu(\sigma \tau)} & =-\frac{1}{2} Q_{\lambda \rho}{ }^{\sigma \tau \mu}+\frac{3}{2} Q_{\lambda \rho}{ }^{(\mu \sigma \tau)} \\
& =-\frac{1}{2} Q_{\lambda \rho}{ }^{\sigma \tau \mu}+\left(\text { terms proportional to } Q_{\gamma \delta}{ }^{\alpha \beta \epsilon}\right. \text { with some pairs of indices contracted), }
\end{aligned}
$$

and furthermore

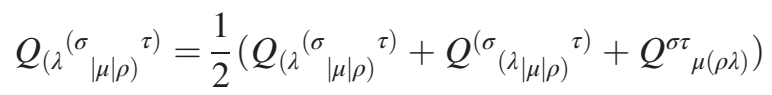

$$
\begin{aligned}
& -\frac{1}{4}\left(Q_{\mu(\rho \lambda)}^{\sigma \tau}+Q^{\sigma \tau}{ }_{(\rho|\mu| \lambda)}+Q_{\rho \lambda \mu}^{\sigma \tau}\right) \\
& +\frac{1}{4} Q_{\rho \lambda \mu}^{\sigma \tau} \text {. }
\end{aligned}
$$

Since the first line of the right-hand side of the above is symmetric under the interchange of the first, second, and fifth indices, and the second line is symmetric under the interchange of the last three indices, they can be eliminated by (4.12) and (4.10). Then

$$
Q_{(\lambda}{ }^{(\sigma|\rho| \rho)}{ }^{\tau)}=\frac{1}{4} Q^{\sigma \tau}{ }_{\lambda \mu}+\left(\text { terms proportional to } Q_{\gamma \delta}{ }^{\alpha \beta \epsilon} \text { with some pairs of indices contracted }\right) .
$$

Using (4.17) and (4.19) for (4.16), we obtain

$$
0=(D+3) Q_{\lambda \rho_{\mu}}^{\sigma \tau}+Q_{\lambda \rho \mu}^{\sigma \tau}+\text { (terms proportional to } Q_{\gamma \delta}^{\alpha \beta \epsilon} \text { with some pairs of indices contracted). }
$$

Subtracting (4.20) with $(\lambda, \rho)$ and $(\sigma, \tau)$ exchanged from (4.20) times $(D+3)$, we obtain

$$
Q_{\lambda \rho} \rho_{\mu}{ }_{\mu}=\text { (terms proportional to } Q_{\gamma \delta}^{\alpha \beta \epsilon} \text { with some pairs of indices contracted). }
$$

Thus we have succeeded in expressing $Q_{\lambda \rho}{ }^{\sigma \tau}{ }_{\mu}$ in terms of tensors with fewer free indices. Contractions of indices of this and other equations lead relations between tensors with some indices contracted. By using them we can simplify (4.21) more. 
From the contractions $(\lambda, \tau)=(\rho, \phi)$ and $(\lambda, \rho)=(\tau, \phi)$ in (4.10), and $(\sigma, \nu)=(\tau, \phi)$ in (4.12),

$$
\begin{aligned}
Q_{\kappa \mu}{ }^{\kappa}{ }_{\sigma} \sigma & =\frac{1}{2}(D+1) Q_{\kappa \mu \sigma}{ }^{\sigma \kappa}, \\
Q_{\kappa \sigma}{ }^{\sigma}{ }_{\mu}{ }^{\kappa} & =\frac{1}{2} Q_{\kappa \mu \sigma}{ }^{\sigma \kappa}+\frac{1}{2} Q_{\kappa}{ }^{\kappa}{ }^{\sigma}{ }_{\mu}, \\
Q_{\kappa \sigma}{ }^{\kappa \sigma}{ }_{\mu} & =Q_{\kappa \mu \sigma}{ }^{\sigma \kappa}-Q_{\kappa}{ }^{\kappa}{ }{ }^{\sigma}{ }^{\sigma}+\frac{1}{2}(D+1) Q_{\kappa}{ }^{\kappa}{ }^{\sigma}{ }^{\sigma}{ }_{\mu} .
\end{aligned}
$$

By using these, $Q_{\kappa \mu}{ }^{\kappa}{ }_{\sigma}{ }^{\sigma}, Q_{\kappa \sigma}{ }^{\sigma}{ }_{\mu}{ }^{\kappa}$, and $Q_{\kappa \sigma}{ }^{\kappa \sigma}{ }_{\mu}$ are expressed in terms of $Q_{\kappa \mu \sigma}{ }^{\sigma \kappa}, Q_{\kappa}{ }^{\kappa}{ }_{\sigma}{ }^{\sigma}$, and $Q_{\kappa}{ }^{\kappa}{ }_{\sigma \mu}{ }^{\sigma}$. Contracting $\lambda$ and $\rho$ in (2.23), renaming $\sigma$ and $\tau$ to $\lambda$ and $\rho$, and adding (4.4) times $D-2$ with $\lambda, \rho$ and $\phi$ symmetrized, we obtain

$$
0=D Q_{\left(\lambda \rho^{\mu \nu}{ }_{\phi)}\right.}-2 Q_{(\lambda \rho \phi)}{ }^{(\mu \nu)}+\left(\text { terms proportional to } Q_{\gamma \delta}{ }^{\alpha \beta \epsilon}\right. \text { with some pairs of indices contracted). }
$$

Contracting $\rho$ and $\phi$ in (2.23) and rewriting $S^{\gamma \delta}{ }_{\alpha \beta}^{\epsilon}$ with no contraction of indices in it by (4.4), we obtain

$0=(D+2) Q_{\lambda\left(\sigma^{\mu \nu}{ }_{\tau}\right)}-2 Q_{\lambda\left(\sigma_{\tau}{ }_{\tau}{ }^{\nu)}\right.}{ }^{\mu}-2 Q_{\lambda}{ }^{(\mu \nu)}{ }_{(\sigma \tau)}+$ (terms proportional to $Q_{\gamma \delta}{ }^{\alpha \beta \epsilon}$ with some pairs of indices contracted).

From the difference of (4.5) and (4.6), and the contraction $\nu=\phi$ in (4.23) minus $\frac{3(2 D-1)}{2(D-2)}$ times the same equation with the indices $\lambda, \rho, \mu$ symmetrized,

$$
\begin{aligned}
& Q_{\lambda \kappa}{ }^{\mu \nu \kappa}=\left(\text { terms proportional to } Q_{\kappa}{ }^{\kappa \alpha \beta \epsilon}, Q_{\gamma \delta \kappa}{ }^{{ }^{k} \epsilon}, Q_{\gamma \kappa}{ }^{\alpha \kappa \epsilon}, Q_{\kappa \alpha \sigma}{ }^{\sigma \kappa}, Q_{\kappa}{ }^{\kappa}{ }_{\sigma}{ }^{\sigma}{ }_{\alpha} \text {, or } Q_{\kappa}{ }^{\kappa}{ }_{\sigma \alpha}{ }^{\sigma}\right) \text {, }
\end{aligned}
$$

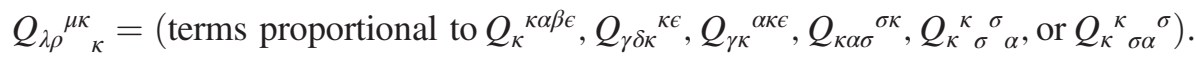

From the contraction $\mu=\nu$ in (4.24) with $\lambda$ renamed $\mu$, plus the contraction $\lambda=\nu$ in (4.24) times $2(D+1)^{2}$,

$$
Q_{\mu \kappa}{ }^{\kappa(\sigma \tau)}=\left(\text { terms proportional to } Q_{\kappa}{ }^{\kappa \alpha \beta \epsilon}, Q_{\gamma \delta \kappa}{ }^{\kappa \epsilon}, Q_{\kappa \alpha \sigma}{ }^{\sigma \kappa}, Q_{\kappa}{ }^{\kappa} \sigma{ }_{\alpha}{ }_{\alpha} \text {, or } Q_{\kappa}{ }^{\kappa}{ }^{\sigma}{ }^{\sigma}\right) \text { ). }
$$

Therefore, with the definition $\phi_{\mu}{ }^{\sigma \tau} \equiv Q_{\mu \kappa}{ }^{k[\sigma]}$,

$$
Q_{\mu \kappa}{ }^{\kappa \sigma \tau}=Q_{\mu \kappa}{ }^{\kappa[\sigma \tau]}+Q_{\mu \kappa}{ }^{\kappa(\sigma \tau)}=\phi_{\mu}{ }^{\sigma \tau}+\left(\text { terms proportional to } Q_{\kappa}{ }^{\kappa \alpha \beta \epsilon}, Q_{\gamma \delta \kappa}{ }^{\kappa \epsilon}, Q_{\kappa \alpha \sigma}{ }^{\sigma \kappa}, Q_{\kappa}{ }^{\kappa}{ }_{\sigma}{ }^{\sigma}{ }_{\alpha} \text {, or } Q_{\kappa}{ }^{\kappa}{ }_{\sigma \alpha}{ }^{\sigma}\right) \text {. }
$$

Contracting $\mu$ and $\sigma$ in (4.28),

$$
Q_{\kappa}{ }^{\kappa \mu \sigma}{ }_{\sigma}=\frac{1}{2} Q^{\kappa \mu \sigma}{ }_{\sigma \kappa}-2 \phi_{\kappa}{ }^{\kappa \mu}+\frac{D}{2} Q_{\kappa}{ }^{\kappa}{ }_{\sigma}{ }^{\sigma \mu} .
$$

From the contraction $\lambda=\tau$ in (4.28), minus $\frac{3}{2} \frac{3 D^{2}+9 D+8}{(D+2)(4 D+1)}$ times the same equation with the indices $\mu, \nu$ and $\sigma$ symmetrized,

$$
Q_{\kappa}{ }^{\kappa \mu \nu \sigma}=\left(\text { terms proportional to } \phi_{\alpha}{ }^{\gamma \delta}, Q_{\gamma \delta \kappa}{ }^{\kappa \epsilon}, \phi_{\kappa}{ }^{\kappa \alpha}, Q_{\kappa \alpha \sigma}{ }^{\sigma \kappa} \text {, or } Q_{\kappa}{ }^{\kappa}{ }_{\sigma}{ }_{\alpha}\right) \text { ). }
$$

Contracting $\mu$ and $\rho$ in (4.21), we obtain an equation which is proportional to $D-3$. Since we consider $D \geq 4$, we can drop this factor (This is the only step at which we use the condition $D \geq 4$.), and

$$
Q_{\lambda \tau \kappa}{ }^{\kappa}{ }_{\sigma}+Q_{\lambda \sigma \kappa}{ }^{\kappa} \tau-2 Q_{\sigma \tau \kappa}{ }^{\kappa} \lambda=\left(\text { terms proportional to } \phi_{\alpha}{ }^{\gamma \delta}, \phi_{\kappa}{ }^{\kappa \alpha}, Q_{\kappa \alpha \sigma}{ }^{\sigma \kappa} \text {, or } Q_{\kappa}{ }^{\kappa}{ }_{\sigma}{ }_{\alpha}{ }_{\alpha}\right. \text { ). }
$$

Then, with the definition $\left.\Phi_{\lambda \sigma \tau} \equiv Q_{(\sigma \tau|\kappa|}{ }^{\kappa} \lambda\right)$,

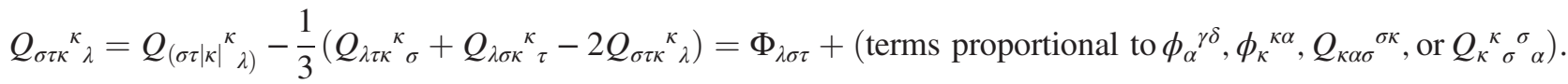


Contracting $\sigma=\tau$ in (4.32)

$$
Q_{\kappa \mu \sigma}{ }^{\sigma \kappa}=-\frac{1}{2} Q_{\kappa}{ }^{\kappa} \sigma^{\sigma}{ }_{\mu}+\frac{3}{2} \Phi^{\kappa}{ }_{\kappa \mu} .
$$

Using (4.22), (4.25), (4.26), (4.28)-(4.30), (4.32), and (4.33), $Q_{\lambda \kappa}{ }^{\mu \nu \kappa}, Q_{\lambda \rho}{ }^{\mu \kappa}{ }_{\kappa}, Q_{\lambda \kappa}{ }^{\kappa \mu \phi}, Q_{\kappa}{ }^{{ }^{\mu} \mu \nu \sigma}$, and $Q_{\lambda \rho \kappa}{ }^{\kappa}{ }_{\phi}$ are expressed in terms of $Q_{\kappa}{ }^{\kappa}{ }_{\sigma}^{\sigma}{ }_{\alpha}, \Phi_{\gamma \delta \epsilon}, \phi_{\alpha}{ }^{\gamma \delta}, \Phi^{\kappa}{ }_{\kappa \alpha}$, and $\phi_{\kappa}{ }^{k \alpha}$. Then from (4.21),

$$
\begin{aligned}
S_{\lambda \rho}{ }^{\mu \nu \phi}= & \text { (terms proportional to } Q_{\kappa}{ }^{\kappa}{ }_{\sigma}{ }^{\sigma} \alpha, \\
& \left.\Phi_{\gamma \delta \varepsilon}, \phi_{\alpha}{ }^{\gamma \delta}, \Phi^{\kappa}{ }_{\kappa \alpha}, \text { or } \phi_{\kappa}{ }^{\kappa \alpha}\right),
\end{aligned}
$$

and it can be confirmed that contractions of this equation reproduce (4.22)-(4.33). In this equation $Q_{\kappa}{ }^{\kappa}{ }_{\sigma}{ }^{\sigma \phi}$ appears only in the combination $Q_{\kappa}{ }^{\kappa}{ }_{\sigma}{ }^{\sigma \phi}-\Phi_{\kappa}{ }^{\kappa \phi}+\frac{8}{3} \phi_{\kappa}{ }^{\kappa \phi}$. To obtain a concise expression of this equation, we define $K^{\phi}, Y_{\lambda \mu \nu}$, and $F_{\lambda \rho}{ }^{\mu}$ as follows:

$$
\begin{aligned}
& K^{\phi}=\frac{3}{2} \frac{1}{(D-1)(D+2)}\left(Q_{\kappa}{ }^{\kappa} \sigma{ }^{\sigma \phi}-\Phi_{\kappa}{ }^{\kappa \phi}+\frac{8}{3} \phi_{\kappa}{ }^{\kappa \phi}\right), \\
& Y_{\lambda \mu \nu}=\frac{4}{D+2} \phi_{[\lambda \mu \nu]}, \\
& F_{\lambda \rho}{ }^{\mu}=\Phi_{\lambda \rho}{ }^{\mu}-\frac{8}{3} \phi_{(\lambda \rho)}{ }^{\mu}-\frac{2}{3}(D+2) \delta_{\left(\lambda^{\mu}\right.} K_{\rho)} \\
& +\frac{1}{3}(2 D+1) G_{\lambda \rho} K^{\mu}
\end{aligned}
$$

Conversely $\phi_{\lambda \mu \nu}$ and $\Phi_{\lambda \rho \mu}$ are given by $K^{\phi}, Y_{\lambda \mu \nu}$, and $F_{\lambda \rho}{ }^{\mu}$ as follows:

$$
\begin{gathered}
\phi_{\lambda \mu \nu}=\frac{1}{4}(D+2) Y_{\lambda \mu \nu}-\frac{1}{2} F_{\lambda[\mu \nu]}+\frac{1}{2}(D+1) G_{\lambda[\mu} K_{\nu]}, \\
\Phi_{\lambda \rho \mu}=F_{(\lambda \rho \mu)}+G_{(\lambda \rho} K_{\mu)} .
\end{gathered}
$$

Then (4.34) is rewritten as follows:

$$
Q_{\lambda \rho}^{\mu \nu \phi}=\delta_{\left(\lambda^{\mu}\right.} \delta_{\rho)}^{\nu} K^{\phi}+\delta_{(\lambda}^{(\mu} Y_{\rho)}^{\nu) \phi}+G^{\phi(\mu} F_{\lambda \rho}{ }^{\nu)} .
$$

Using this, (4.8) is simplified further. Let us define $H_{\lambda}^{\mu \nu}$ as follows:

$$
H_{\lambda}^{\mu \nu}=\frac{2}{D+1}\left(S_{\kappa \lambda}{ }^{\mu \nu \kappa}-\delta_{\lambda}{ }^{(\mu} K^{\nu)}\right)
$$

then

$$
S_{\lambda \rho}^{\mu \nu \phi}=\delta_{\left(\lambda^{\mu}\right.}{ }^{\mu} \delta_{\rho)}{ }^{\nu} K^{\phi}+\delta_{(\lambda}{ }^{(\mu} Y_{\rho)}{ }^{\nu) \phi}+\delta^{\phi}{ }_{(\lambda} H^{\mu \nu}{ }_{\rho)} .
$$

It is not difficult to confirm that (4.40) and (4.42) solve (2.23). Therefore (4.40) and (4.42) give the general solution to (2.23).

\section{Analysis of (2.24)}

Next let us solve (2.24). Contracting $\tau$ and $\phi$, $s_{\lambda \rho}{ }^{\mu \nu}=s_{\kappa}{ }^{\kappa \mu \nu} G_{\lambda \rho}+$ (terms proportional to $q_{\gamma \delta}{ }^{\alpha \beta}$

$$
\text { or } \left.\nabla_{\zeta} S_{\gamma \delta}^{\alpha \beta \epsilon}\right) \text {. }
$$

Contracting $\lambda$ and $\rho$ in this equation,

$s_{\kappa}{ }^{\kappa \mu \nu}=\left(\right.$ terms proportional to $q_{\gamma \delta}^{\alpha \beta}$ or $\left.\nabla_{\zeta} S_{\gamma \delta}{ }^{\alpha \beta \epsilon}\right)$.

Using this and (4.43), we can express $s_{\lambda \rho}{ }^{\mu \nu}$ in terms of other tensors:

$s_{\lambda \rho}^{\mu \nu}=\left(\right.$ terms proportional to $q_{\gamma \delta}^{\alpha \beta}$ or $\left.\nabla_{\zeta} S_{\gamma \delta}^{\alpha \beta \epsilon}\right)$.

Contracting $\lambda$ and $\rho$ in (2.24), we obtain the following equation similar to (but different from) (4.43).

$$
\begin{aligned}
s_{\lambda \rho}{ }^{\mu \nu}= & s_{\kappa}{ }^{\mu \nu \nu} G_{\lambda \rho}+\left(\text { terms proportional to } q_{\gamma \delta}{ }^{\alpha \beta}\right. \\
& \text { or } \left.\nabla_{\zeta} S_{\gamma \delta}{ }^{\alpha \beta \epsilon}\right) .
\end{aligned}
$$

Taking the difference of (4.43) and (4.46), we obtain the following equation which does not contain $s_{\gamma \delta}^{\alpha \beta}$ :

$$
\begin{aligned}
& q^{\tau \phi \mu \nu}=q^{\tau \phi \kappa}{ }_{\kappa} G^{\mu \nu}+\frac{1}{D-2}\left[q_{\kappa}{ }^{\kappa \mu \nu} G^{\tau \phi}+q_{\kappa}{ }^{\kappa \tau \phi} G^{\mu \nu}\right. \\
& -q_{\kappa}{ }^{\kappa \tau(\mu} G^{\nu) \phi}-q_{\kappa}{ }^{\kappa \phi(\mu} G^{\nu) \tau} \\
& \left.+q_{\kappa}{ }^{\kappa} \sigma_{\sigma} \sigma\left(G^{\tau(\mu} G^{\nu) \phi}-G^{\mu \nu} G^{\tau \phi}\right)\right] \\
& \left.+ \text { (terms proportional to } \nabla_{\zeta} S_{\gamma \delta}^{\alpha \beta \epsilon}\right) \text {. }
\end{aligned}
$$

Contracting $\mu$ and $\nu$ in this equation, we obtain $q^{\tau \phi{ }_{k}}{ }_{\kappa}$ expressed by $q_{\kappa}{ }^{\kappa \alpha \beta}$ and terms proportional to $\nabla_{\zeta} S_{\gamma \delta}{ }^{\alpha \beta \epsilon}$. Using it (4.47) can be simplified further:

$q^{\tau \phi \mu \nu}=\left(\right.$ terms proportional to $q_{\kappa}{ }^{\alpha \beta \beta}$ or $\left.\quad \nabla_{\zeta} S_{\gamma \delta}{ }^{\alpha \beta \epsilon}\right)$.

From (4.45), (4.48) and (4.42),

$q_{\lambda \rho}^{\mu \nu}=\left(\right.$ terms proportional to $q_{\kappa}{ }^{\alpha \beta \beta}, \nabla_{\epsilon} K^{\alpha}$, or $\left.\nabla_{\epsilon} Y_{\alpha \beta \gamma}\right)$,

$$
\begin{aligned}
s_{\lambda \rho}{ }^{\mu \nu}= & \left(\text { terms proportional to } q_{\kappa}{ }^{\kappa \alpha \beta}, \nabla_{\epsilon} K^{\alpha},\right. \\
& \left.\nabla_{\epsilon} Y_{\alpha \beta \gamma}, \text { or } \nabla_{\epsilon} H^{\alpha \beta}{ }_{\gamma}\right) .
\end{aligned}
$$

Then (2.24) is rewritten as follows by (4.49), (4.50), and (4.42): 
$0=\left(\right.$ terms proportional to $q_{\kappa}{ }^{\kappa \alpha \beta}, \nabla_{\epsilon} K^{\alpha}$, or $\left.\nabla_{\epsilon} Y_{\alpha \beta \gamma}\right)$.

From the contraction $(\mu, \rho)=(\nu, \tau)$ in (4.51),

$$
\nabla^{\kappa} Y_{\kappa \mu \nu}=0,
$$

and from the contraction $(\nu, \lambda)=(\rho, \tau)$ in $(4.51)$,

$q_{\kappa}{ }^{\kappa \mu \nu}=\frac{1}{D} G^{\mu \nu} q_{\kappa}{ }^{\kappa}{ }_{\sigma} \sigma-\nabla^{\mu} K^{\nu}-\nabla^{\nu} K^{\mu}+\frac{2}{D} G^{\mu \nu} \nabla_{\kappa} K^{\kappa}$.

Then from the contraction $(\nu, \mu)=(\rho, \tau)$ in (4.51),

$$
\nabla^{\mu} K^{\nu}+\nabla^{\nu} K^{\mu}=\frac{2}{D} G^{\mu \nu} \nabla_{\kappa} K^{\kappa},
$$

which means that $K^{\mu}$ is a conformal Killing vector. Contracting $\nu$ and $\rho$ in (4.51),

$$
0=\nabla_{\tau} Y_{\lambda \mu \phi}+\nabla_{\phi} Y_{\lambda \mu \tau}
$$

which means that $\nabla_{\tau} Y_{\lambda \mu \phi}$ is antisymmetric in the interchange of all of four indices, i.e., $Y_{\lambda \mu \nu}$ is a Killing-Yano 3 -form. This implies (4.52), and from (4.53),

$$
q_{\kappa}{ }^{\kappa \mu \nu}=\tilde{c} G^{\mu \nu},
$$

where $\tilde{c}=\frac{1}{D} q_{\kappa}{ }^{\kappa}{ }_{\sigma}{ }^{\sigma}$. Then (4.49) and (4.50) are rewritten as follows:

$$
\begin{aligned}
& q_{\lambda \rho}{ }^{\mu \nu}=\delta_{(\lambda}{ }^{(\mu}\left(\nabla_{\rho)} K^{\nu)}-\nabla^{\nu)} K_{\rho)}\right)+\delta_{\left(\lambda^{\mu} \delta_{\rho)}{ }^{\nu} \tilde{c},\right.}
\end{aligned}
$$

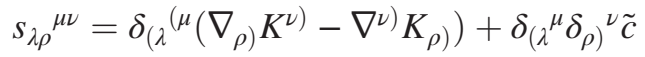

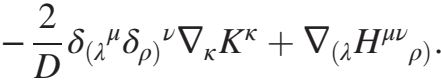

It can be confirmed that these solve (2.24), and therefore these give the general solution to (2.24).

\section{Analysis of (2.25)}

Next let us analyze (2.25). Rewriting (2.25) by (4.40), (4.42), (4.57), and (4.58), and using (A2), (A5), and Bianchi identity $R_{[\rho \mu \nu]}^{\lambda}=0$, we obtain

$$
\begin{aligned}
& 0=\frac{1}{4}\left(\nabla^{\phi} \nabla_{(\lambda}-\nabla_{(\lambda} \nabla^{\phi}\right) H_{\rho \nu}^{\mu \nu} \\
& +\frac{3}{8}\left(\nabla^{\kappa} \nabla^{\phi}-\nabla^{\phi} \nabla^{\kappa}\right) H^{\mu \nu}{ }_{\kappa}+\frac{1}{2} \delta_{(\lambda}{ }^{\phi}\left(\nabla_{\rho)} \nabla^{\kappa}-\nabla^{\kappa} \nabla_{\rho)}\right) H^{\mu \nu}{ }_{\kappa} \\
& +\frac{1}{24} \delta_{(\lambda}{ }^{(\mu} R^{|\kappa \sigma \phi| \nu)} Y_{\rho) \kappa \sigma}+\frac{1}{24} \delta_{(\lambda}^{(\mu} R^{|\kappa \sigma \phi|}{ }_{\rho)} Y_{\kappa \sigma}^{\nu)} \\
& -\frac{1}{24} \delta_{(\lambda}^{(\mu} R^{|\kappa \sigma| \nu) \phi} Y_{\rho) \kappa \sigma}-\frac{1}{24} \delta_{(\lambda}^{(\mu} R_{\rho)}^{|\kappa \sigma| \nu)} Y_{\kappa \sigma}{ }_{\kappa \sigma} \\
& +\frac{1}{4} G^{\phi(\mu} R^{|\kappa \sigma| \nu)}{ }_{(\lambda} Y_{\rho) \kappa \sigma}+\frac{1}{8} \delta_{(\lambda}^{\phi} R_{\rho \rho}^{\kappa \sigma}{ }_{\rho}^{(\mu} Y_{\kappa \sigma}^{\nu)} \\
& -\frac{1}{8} G^{\mu \nu} R^{\kappa \sigma \phi}{ }_{(\lambda} Y_{\rho) \kappa \sigma}-\frac{1}{16} G_{\lambda \rho} R^{\kappa \sigma \phi(\mu} Y^{\nu}{ }_{\kappa \sigma}
\end{aligned}
$$

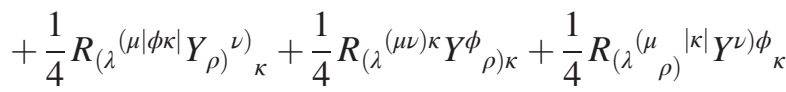

$$
\begin{aligned}
& +\left(\text { terms proportional to } \partial_{\alpha} \tilde{c}, \partial_{\alpha}\left(\nabla_{\kappa} K^{\kappa}\right), R_{\gamma}^{\kappa} Y_{\kappa \alpha \beta}, \Lambda F_{\gamma \delta}{ }^{\alpha} \text {, or } \Lambda H^{\alpha \beta}{ }_{\gamma}\right) \\
& + \text { (terms proportional to products of } F_{\gamma \delta}{ }^{\alpha} \text { and Ricci scalar, Ricci tensor, or Riemann tensor) } \\
& + \text { (terms proportional to products of } H^{\alpha \beta}{ }_{\gamma} \text { and Ricci scalar, Ricci tensor, or Riemann tensor). }
\end{aligned}
$$

The first two lines of the above can be rewritten by using

$$
\begin{aligned}
\left(\nabla_{\lambda} \nabla_{\rho}-\nabla_{\rho} \nabla_{\lambda}\right) H^{\mu \nu}{ }_{\phi}= & R_{\lambda \rho}{ }^{\mu}{ }_{\kappa} H^{\kappa \nu}{ }_{\phi}+R_{\lambda \rho}{ }^{\nu} H^{\mu \kappa}{ }_{\phi} \\
& +R_{\lambda \rho \phi \kappa} H^{\mu \nu \kappa},
\end{aligned}
$$

and by applying (A7) to the third line, the fourth line, and each term in the fifth and sixth lines of the above, the Riemann tensors in those terms can be rewritten in terms of Ricci tensor. Furthermore the seventh line vanishes by (A6).
After these simplifications, we obtain the followings by contracting two pairs of indices in (4.59) in various ways: $\partial_{\mu} \tilde{c}=\left(\right.$ terms proportional to $F_{\mu \kappa}{ }^{\kappa}, H^{\kappa}{ }_{\kappa \mu}$, or $\left.R_{\kappa \sigma} H^{\kappa \sigma}{ }_{\mu}\right)$,

$\partial_{\mu}\left(\nabla_{\kappa} K^{\kappa}\right)=\left(\right.$ terms proportional to $F_{\kappa}{ }^{\kappa \mu}, F_{\mu \kappa}{ }^{\kappa}, H^{\kappa}{ }_{\kappa \mu}, H^{\mu \kappa}{ }_{\kappa}$,

$$
\left.R_{\kappa \sigma} H^{\kappa \sigma}{ }_{\mu} \text {, or } R_{\kappa \sigma} H^{\mu \kappa \sigma}\right) \text {, }
$$


$R_{\kappa \sigma} F^{\kappa \sigma \mu}=\left(\right.$ terms proportional to $F_{\kappa}{ }^{\kappa \mu}, H^{\mu \kappa}{ }_{\kappa}$, or $\left.R_{\kappa \sigma} H^{\mu \kappa \sigma}\right)$,

$R_{\kappa \sigma} F_{\mu}{ }^{\kappa \sigma}=\left(\right.$ terms proportional to $F_{\mu \kappa}{ }^{\kappa}, H^{\kappa}{ }_{\kappa \mu}$, or $\left.R_{\kappa \sigma} H^{\kappa \sigma}{ }_{\mu}\right)$,

$R_{\mu \kappa} F_{\kappa \sigma}{ }^{\sigma}=$ (terms proportional to $F_{\mu \kappa}{ }^{\kappa}, H^{\mu \kappa}{ }_{\kappa}$, or $R_{\mu \kappa} H^{\kappa \sigma}{ }_{\sigma}$ ),

$R_{\mu \kappa} F_{\sigma}{ }^{\sigma \kappa}=$ (terms proportional to $F_{\kappa}{ }^{\kappa \mu}, F_{\mu \kappa}{ }^{\kappa}, H^{\kappa}{ }_{\kappa \mu}, H^{\mu \kappa}{ }_{\kappa}$,

$$
\left.R_{\kappa \sigma} H^{\kappa \sigma}{ }_{\mu}, R_{\kappa \sigma} H^{\mu \kappa \sigma} \text {, or } R_{\mu \kappa} H_{\sigma}^{\sigma \kappa}\right) \text {. }
$$

Then from (4.59) minus 3 times (4.59) with the indices $\mu, \nu$, $\phi$ symmetrized,

$$
\begin{aligned}
& \left(R^{\mu \nu}-\frac{1}{2} G^{\mu \nu} R+\Lambda G^{\mu \nu}\right) F_{\lambda \rho}{ }^{\phi} \\
& =\text { (terms proportional to } \Lambda F_{\kappa}{ }^{\kappa \alpha}, \Lambda F_{\alpha \kappa}{ }^{\kappa}, R F_{\kappa}{ }^{\kappa \alpha}, R F_{\alpha \kappa}{ }^{{ }} \text {, } \\
& \Lambda H^{\kappa}{ }_{\kappa \alpha}, \Lambda H^{\alpha \kappa}{ }_{\kappa}, \Lambda H^{\alpha \beta \gamma}, R H^{\kappa}{ }^{\kappa}{ }_{\alpha \alpha}, R H^{\alpha{ }_{k}}, R H^{\alpha \beta \gamma} \text {, } \\
& \left.R_{\kappa \sigma} H^{\kappa \sigma \alpha}, R_{\kappa \sigma} H^{\alpha \kappa \sigma}, R^{\alpha \beta} H_{\gamma \delta}{ }^{\epsilon} \text {, or } R_{\gamma}^{\kappa} Y_{\kappa \alpha \beta}\right) \text {. }
\end{aligned}
$$

It can be confirmed that this, (4.61), and (4.62) solve (4.59), and reproduce (4.63)-(4.66). Therefore (4.67), (4.61), and (4.62) are equivalent to (2.25), and no simpler equation is derived from them.

Note that Riemann tensor (with no indices contracted) does not appear in (4.61), (4.62), and (4.67), and in fact they are in the forms that Ricci tensor and $\Lambda$ appear only in the form of the background equation of motion. The results are summarized in (3.6), (3.7), and (3.8).

\section{E. Analysis of (2.26)}

Next let us analyze (2.26). Rewriting (2.26) by (4.40), (4.42), (4.57), and (4.58), and using (A1), (A2), (A4), and Bianchi identities

$$
\begin{aligned}
R_{[\rho \mu \nu]}^{\lambda} & =0, \quad \nabla_{[\lambda} R_{\mu \nu] \sigma \tau}=0, \\
\nabla^{\kappa} R_{\kappa \lambda \mu \nu} & =2 \nabla_{[\mu} R_{\nu] \lambda}, \quad \nabla^{\kappa} R_{\kappa \mu}=\frac{1}{2} \partial_{\mu} R,
\end{aligned}
$$

we obtain

$$
\begin{aligned}
0= & \frac{1}{4}\left[\nabla_{(\lambda}\left(\nabla_{\rho)} \nabla_{\kappa}-\nabla_{|\kappa|} \nabla_{\rho)}\right)+\left(\nabla_{(\lambda} \nabla_{|\kappa|}-\nabla_{\kappa} \nabla_{(\lambda}\right) \nabla_{\rho)}\right] H^{\mu \nu \kappa} \\
+ & \left.\frac{1}{4} \nabla^{\kappa}\left(\nabla_{\kappa} \nabla_{(\lambda}-\nabla_{(\lambda} \nabla_{|\kappa|}\right) H^{\mu \nu}{ }_{\rho}\right) \\
+ & \frac{1}{8} G_{\lambda \rho}\left[\left(\nabla_{\kappa} \nabla_{\sigma}-\nabla_{\sigma} \nabla_{\kappa}\right) \nabla^{\sigma}+2 \nabla^{\sigma}\left(\nabla_{\kappa} \nabla_{\sigma}-\nabla_{\sigma} \nabla_{\kappa}\right)\right] H^{\mu \nu \sigma} \\
+ & \frac{1}{4} \nabla^{(\mu} R^{\nu)}{ }_{(\lambda}{ }^{\kappa \sigma} Y_{\rho) \kappa \sigma}+\frac{1}{8} \nabla_{(\lambda} R_{\rho)}{ }^{(\mu|\kappa \sigma|} Y^{\nu)}{ }_{\kappa \sigma} \\
+ & \frac{3}{8} R^{\kappa \sigma}{ }_{(\lambda}{ }^{(\mu} \nabla_{\mid \kappa} Y_{\sigma \mid \rho)}{ }^{\nu)} \\
- & \frac{1}{8} G_{\lambda \rho} G^{\mu \nu} K^{\kappa} \nabla_{\kappa} R+\frac{1}{4} \delta_{(\lambda}{ }^{\mu} \delta_{\rho)}{ }^{\nu} K^{\kappa} \nabla_{\kappa} R \\
+ & \frac{1}{4} G_{\lambda \rho} K^{\kappa} \nabla_{\kappa} R^{\mu \nu}+\frac{1}{4} G^{\mu \nu} K^{\kappa} \nabla_{\kappa} R_{\lambda \rho}-\delta_{(\lambda}{ }^{\mu} K^{|\kappa|} \nabla_{|\kappa|} R_{\rho)}{ }^{\nu)} \\
- & \frac{1}{4} G_{\lambda \rho}\left(R_{\kappa}{ }^{\mu} \nabla^{[\kappa} K^{\nu]}+R_{\kappa}{ }^{\nu} \nabla^{[\kappa} K^{\mu]}\right)-\frac{1}{4} G^{\mu \nu}\left(R^{\kappa}{ }_{\lambda} \nabla_{[\kappa} K_{\rho]}+R^{\kappa}{ }_{\rho} \nabla_{[\kappa} K_{\lambda]}\right) \\
+ & \frac{1}{2} \delta_{(\lambda}{ }^{\mu} R_{\rho) \kappa} \nabla^{[\kappa} K^{\nu]}+\frac{1}{2} \delta_{(\lambda}{ }^{\nu} R_{\rho) \kappa} \nabla^{[\kappa} K^{\mu]}+\frac{1}{2} \delta_{\lambda}{ }^{(\mu} R^{\nu) \kappa} \nabla_{[\kappa} K_{\rho]}+\frac{1}{2} \delta_{\rho}{ }^{(\mu} R^{\nu) \kappa} \nabla_{[\kappa} K_{\lambda]} \\
+ & \left(\text { terms proportional to } \nabla_{\alpha} \nabla_{\beta} \tilde{c}, \Lambda \nabla_{\epsilon} H^{\alpha \beta \gamma}, R_{\gamma \delta \zeta \eta} \nabla_{\epsilon} H^{\alpha \beta \theta}, \nabla_{\epsilon} R_{\gamma \delta \zeta \eta} H^{\alpha \beta \theta},\right. \\
& \quad \nabla_{\epsilon} R H^{\alpha \beta \gamma}, R_{\gamma \delta} \nabla_{\epsilon} H^{\alpha \beta \theta}, \nabla_{\epsilon} R_{\gamma \delta} H^{\alpha \beta \theta}, \\
& \left.R \nabla_{\kappa} K^{\kappa}, \Lambda \nabla_{\kappa} K^{\kappa}, R_{\alpha \beta} \nabla_{\kappa} K^{\kappa}, \nabla_{\alpha} \nabla_{\beta} \nabla_{\kappa} K^{\kappa}, \nabla_{\epsilon} R_{\zeta \eta} Y_{\alpha \beta \gamma}, \nabla_{\epsilon} R Y_{\alpha \beta \gamma}\right) .
\end{aligned}
$$

The first, second and third lines of the above can be simplified by replacing the commutators of covariant derivatives by Riemann tensors, and the fourth line is rewritten as follows: 


$$
\begin{aligned}
& \frac{1}{4} \nabla^{(\mu} R^{\nu)}{ }_{(\lambda}{ }^{\kappa \sigma} Y_{\rho) \kappa \sigma}+\frac{1}{8} \nabla_{(\lambda} R_{\rho)}{ }^{(\mu|\kappa \sigma|} Y^{\nu)}{ }_{\kappa \sigma} \\
& =\nabla^{(\mu}\left[\frac{1}{4} R^{|\kappa \sigma| \nu)}{ }_{(\lambda} Y_{\rho) \kappa \sigma}\right]+\nabla_{(\lambda}\left[\frac{1}{8} R^{\kappa \sigma}{ }_{\rho)}^{(\mu} Y^{\nu)}{ }_{\kappa \sigma}\right] \\
& -\frac{1}{4} R^{(\nu}{ }_{\left(\lambda^{|\kappa \sigma|} \nabla^{\mu)}\right.} Y_{\rho) \kappa \sigma}-\frac{1}{8} R_{(\rho}{ }^{(\mu|\kappa \sigma|} \nabla_{\lambda)} Y^{\nu)}{ }_{\kappa \sigma} .
\end{aligned}
$$

The first line of the above can be simplified by (A7), and the second line can be simplified by using (A4):

$$
\begin{aligned}
& \frac{1}{4} \nabla^{(\mu} R^{\nu)}{ }_{(\lambda}{ }^{\kappa \sigma} Y_{\rho) \kappa \sigma}+\frac{1}{8} \nabla_{(\lambda} R_{\rho)}{ }^{(\mu|\kappa \sigma|} Y^{\nu)}{ }_{\kappa \sigma} \\
& \left.=\nabla^{(\mu}\left[-\frac{1}{2} R^{|\kappa|}{ }_{(\lambda} Y^{\nu)} \rho\right) \kappa\right]+\nabla_{(\lambda}\left[-\frac{1}{4} R^{\kappa(\mu} Y_{\rho)^{\nu}}{ }_{\kappa}{ }_{\kappa}\right] \\
& -\frac{1}{4} R^{\left({ }^{\nu}{ }_{(\lambda}{ }^{|\kappa \sigma|} \nabla_{\mid \kappa} Y_{\sigma \mid}{ }^{\mu)}{ }_{\rho)}\right.}-\frac{1}{8} R_{(\rho}{ }^{(\mu|\kappa \sigma|} \nabla_{\mid \kappa} Y_{\sigma \mid \lambda)}{ }^{\nu)} \\
& =-\frac{1}{2} \nabla^{(\mu} R^{|\kappa|}{ }_{(\lambda} Y^{\nu)}{ }_{\rho) \kappa}-\frac{1}{4} \nabla_{(\lambda} R^{\kappa(\mu} Y_{\rho)^{\nu}{ }_{\kappa}{ }_{\kappa}} \\
& \left.-\frac{3}{8} R_{(\lambda}{ }^{(\mu|\kappa \sigma|} \nabla_{\mid \kappa} Y_{\sigma \mid \rho)}\right)^{\nu)} \text {. }
\end{aligned}
$$

The last term of the above cancels the fifth line of (4.69). The sixth and seventh line are simplified by the following relations derived from (A3):

$$
\begin{aligned}
K^{\kappa} \nabla_{\kappa} R_{\mu \nu}= & -\nabla_{\mu} K^{\kappa} R_{\kappa \nu}-\nabla_{\nu} K^{\kappa} R_{\kappa \mu} \\
& -\frac{D-2}{D} \nabla_{\mu} \nabla_{\nu} \nabla_{\kappa} K^{\kappa}-\frac{1}{D} G_{\mu \nu} \nabla^{\sigma} \nabla_{\sigma} \nabla_{\kappa} K^{\kappa} \\
= & -\nabla_{[\mu} K_{\kappa]} R^{\kappa}{ }_{\nu}-\nabla_{[\nu} K_{\kappa]} R^{\kappa}{ }_{\mu}-\frac{2}{D} \nabla_{\kappa} K^{\kappa} R_{\mu \nu} \\
& -\frac{D-2}{D} \nabla_{\mu} \nabla_{\nu} \nabla_{\kappa} K^{\kappa}-\frac{1}{D} G_{\mu \nu} \nabla^{\sigma} \nabla_{\sigma} \nabla_{\kappa} K^{\kappa}, \\
K^{\kappa} \partial_{\kappa} R= & -\frac{2(D-1)}{D} \nabla^{\sigma} \nabla_{\sigma} \nabla_{\kappa} K^{\kappa}-\frac{2}{D} \nabla_{\kappa} K^{\kappa} R,
\end{aligned}
$$

where we used

$$
\nabla_{\mu} K_{\nu}=\nabla_{[\mu} K_{\nu]}+\frac{1}{D} G_{\mu \nu} \nabla_{\kappa} K^{\kappa}
$$

Then terms proportional to $\nabla_{[\mu} K_{\nu]}$ from the seventh line cancel the eighth and ninth lines of (4.69).

(4.69) is further simplified by (3.6) and (3.7). Then from the contraction $(\mu, \lambda)=(\nu, \rho)$ and $(\mu, \nu)=(\lambda, \rho)$ in (4.69),

$$
\begin{aligned}
\nabla_{\kappa} H^{\kappa \sigma \tau} R_{\sigma \tau}= & \text { (terms proportional to } \nabla_{\kappa} F_{\sigma}{ }^{\sigma \kappa} \Lambda, \nabla_{\kappa} F^{\kappa \sigma}{ }_{\sigma} \Lambda, \nabla_{\kappa} F_{\sigma}{ }^{\sigma \kappa} R, \nabla_{\kappa} F^{\kappa \sigma}{ }_{\sigma} R, F^{\kappa \sigma}{ }_{\sigma} \nabla_{\kappa} R, F_{\sigma}{ }^{\sigma \kappa} \nabla_{\kappa} R, \\
& \nabla_{\kappa} H_{\sigma}{ }^{\sigma \kappa} \Lambda, \nabla_{\kappa} H^{\kappa \sigma}{ }_{\sigma} \Lambda, \nabla_{\kappa} H_{\sigma}{ }^{\sigma \kappa} R, \nabla_{\kappa} H^{\kappa \sigma}{ }_{\sigma} R, H^{\kappa \sigma}{ }_{\sigma} \nabla_{\kappa} R, H_{\sigma}{ }^{\sigma \kappa} \nabla_{\kappa} R, \\
& \left.\nabla_{\kappa} H_{\sigma \tau}{ }^{\tau} R^{\kappa \sigma}, H^{\sigma \tau \kappa} \nabla_{\sigma} R_{\tau \kappa}, \nabla_{\kappa} H^{\tau}{ }_{\tau \sigma} R^{\kappa \sigma}, \Lambda \nabla_{\kappa} K^{\kappa}\right),
\end{aligned}
$$

$\nabla_{\kappa} H^{\sigma \tau \kappa} R_{\sigma \tau}=$ (terms proportional to $\nabla_{\kappa} F^{\kappa \sigma}{ }_{\sigma} \Lambda, \nabla_{\kappa} F^{\kappa \sigma}{ }_{\sigma} R, F^{\kappa \sigma}{ }_{\sigma} \nabla_{\kappa} R, \nabla_{\kappa} H_{\sigma}{ }^{\sigma \kappa} \Lambda, \nabla_{\kappa} H^{\kappa \sigma}{ }_{\sigma} \Lambda, \nabla_{\kappa} H_{\sigma}{ }^{\sigma \kappa} R, \nabla_{\kappa} H^{\kappa \sigma}{ }_{\sigma} R, H_{\sigma}{ }^{\sigma \kappa} \nabla_{\kappa} R$,

$$
\left.\nabla_{\kappa} H_{\sigma \tau}{ }^{\tau} R^{\kappa \sigma}, H^{\sigma \tau \kappa} \nabla_{\kappa} R_{\sigma \tau}, \nabla_{\kappa} H_{\tau \sigma}^{\tau} R^{\kappa \sigma}\right) .
$$

Then using the above, from the contraction $\mu=\nu$ and $\lambda=\rho$ in (4.69),

$\nabla_{(\mu} H^{\kappa \sigma}{ }_{\nu)} R_{\kappa \sigma}=$ (terms proportional to $\Lambda \nabla_{\kappa} F^{\kappa \sigma}{ }_{\sigma}, \Lambda \nabla_{\alpha} F_{\beta \kappa}{ }^{\kappa}, R \nabla_{\kappa} F^{\kappa \sigma}{ }_{\sigma}, R \nabla_{\alpha} F_{\beta \kappa}{ }^{\kappa}, F^{\kappa \sigma}{ }_{\sigma} \nabla_{\kappa} R, F_{\beta \kappa}{ }^{\kappa} \nabla_{\alpha} R, \Lambda \nabla^{\alpha} H_{\kappa}{ }^{\kappa \beta}, \Lambda \nabla_{\kappa} H_{\sigma}{ }^{\sigma \kappa}$, $\Lambda \nabla_{\kappa} H^{\kappa \sigma}{ }_{\sigma}, \Lambda \nabla_{\kappa} H^{\alpha \beta \kappa}, R \nabla^{\alpha} H_{\kappa}{ }^{\kappa \beta}, R \nabla_{\kappa} H_{\sigma}{ }^{\sigma \kappa}, R \nabla_{\kappa} H^{\kappa \sigma}{ }_{\sigma}, R \nabla_{\kappa} H^{\alpha \beta \kappa}, H_{\sigma}{ }^{\sigma \kappa} \nabla_{\kappa} R, H_{\kappa}{ }^{\kappa \alpha} \nabla^{\beta} R, H^{\alpha \beta \kappa} \nabla_{\kappa} R$,

$$
\left.R_{\alpha \beta} \nabla_{\kappa} H_{\sigma}{ }^{\sigma \kappa}, R_{\kappa}{ }^{\alpha} \nabla^{\kappa} H_{\sigma}{ }^{\sigma \beta}, R_{\kappa \sigma} \nabla^{\kappa} H_{\tau}^{\tau \sigma}, R_{\kappa \sigma} \nabla^{\kappa} H^{\sigma \tau}{ }_{\tau}, R_{\kappa \sigma} \nabla^{\kappa} H^{\alpha \beta \sigma}, H_{\sigma}{ }^{\sigma \kappa} \nabla_{\kappa} R_{\alpha \beta}, H^{\kappa \sigma \alpha} \nabla^{\beta} R_{\kappa \sigma}, \nabla_{\kappa} R_{\sigma \alpha} Y_{\beta}{ }^{\kappa \sigma}\right) \text {, }
$$

$\nabla_{(\mu} H_{\nu}{ }^{\kappa \sigma} R_{\kappa \sigma}=$ (terms proportional to $\Lambda \nabla^{\alpha} F_{\kappa}{ }^{\kappa \beta}, \Lambda \nabla_{\kappa} F^{\kappa \sigma}{ }_{\sigma}, R \nabla^{\alpha} F_{\kappa}{ }^{\kappa \beta}, R \nabla_{\kappa} F^{\kappa \sigma}{ }_{\sigma}, F_{\kappa}{ }^{\kappa \alpha} \nabla^{\beta} R, F^{\kappa \sigma}{ }_{\sigma} \nabla_{\kappa} R, \Lambda \nabla^{\alpha} H^{\beta \kappa}{ }_{\kappa}, \Lambda \nabla^{\alpha} H_{\kappa}{ }^{\kappa \beta}$,

$$
\begin{aligned}
& \Lambda \nabla_{\kappa} H_{\sigma}{ }^{\sigma \kappa}, \Lambda \nabla_{\kappa} H^{\kappa \sigma}{ }_{\sigma}, \Lambda \nabla_{\kappa} H^{\alpha \beta \kappa}, R \nabla^{\alpha} H^{\beta \kappa}{ }_{\kappa}, R \nabla^{\alpha} H_{\kappa}{ }^{\kappa \beta}, R \nabla_{\kappa} H_{\sigma}{ }^{\sigma \kappa}, R \nabla_{\kappa} H^{\kappa \sigma}{ }_{\sigma}, R \nabla_{\kappa} H^{\alpha \beta \kappa}, H_{\sigma}{ }^{\sigma \kappa} \nabla_{\kappa} R, \\
& H^{\alpha \kappa}{ }_{\kappa} \nabla^{\beta} R, H^{\alpha \beta \kappa} \nabla_{\kappa} R, R_{\alpha \beta} \nabla_{\kappa} H_{\sigma}{ }^{\sigma \kappa}, R_{\kappa}{ }^{\alpha} \nabla^{\kappa} H_{\sigma}{ }^{\sigma \beta}, R_{\kappa \sigma} \nabla^{\kappa} H^{\sigma \tau}{ }_{\tau}, R_{\kappa \sigma} \nabla^{\kappa} H_{\tau}{ }^{\tau \sigma}, R_{\kappa \sigma} \nabla^{\kappa} H^{\alpha \beta \sigma}, H_{\sigma}{ }^{\sigma \kappa} \nabla_{\kappa} R_{\alpha \beta}, \\
& \left.H^{\alpha \kappa \sigma} \nabla^{\beta} R_{\kappa \sigma}, \nabla_{\kappa} R_{\sigma \alpha} Y_{\beta}{ }^{\kappa \sigma}, \Lambda \nabla_{\kappa} K^{\kappa}\right) .
\end{aligned}
$$

Equations (4.69) simplified by the above, (4.77), and (4.78) are equivalent to (4.69). They can be rewritten in the form given in (3.9), (3.10), and (3.11). No simpler relation can be derived from them.

\section{ACKNOWLEDGMENTS}

I would like to thank T. Houri for correspondence. 


\section{APPENDIX: CONFORMAL KILLING VECTORS AND KILLING-YANO TENSORS}

A conformal Killing vector $K^{\mu}$ is defined by

$$
\nabla_{\mu} K_{\nu}+\nabla_{\nu} K_{\mu}=\frac{2}{D} g_{\mu \nu} \nabla_{\lambda} K^{\lambda}
$$

If the right-hand side vanishes, $K^{\mu}$ is a Killing vector. From this equation we can show the following:

$$
\nabla_{\mu} \nabla_{\nu} K_{\lambda}=R_{\lambda \nu \mu \rho} K^{\rho}+\frac{1}{D}\left[g_{\lambda \mu} \partial_{\nu}\left(\nabla_{\rho} K^{\rho}\right)+g_{\lambda \nu} \partial_{\mu}\left(\nabla_{\rho} K^{\rho}\right)-g_{\mu \nu} \partial_{\lambda}\left(\nabla_{\rho} K^{\rho}\right)\right],
$$

and the Lie derivative of Riemann tensor along $K^{\mu}$ is given by

$$
\mathcal{L}_{K} R_{\rho \mu \nu}^{\lambda}=\frac{1}{D}\left(\delta_{\nu}{ }^{\lambda} \delta_{\rho}{ }^{\sigma}-g_{\rho \nu} g^{\lambda \sigma}\right) \nabla_{\mu} \nabla_{\sigma} \nabla_{\tau} K^{\tau}-\frac{1}{D}\left(\delta_{\mu}{ }^{\lambda} \delta_{\rho}{ }^{\sigma}-g_{\rho \mu} g^{\lambda \sigma}\right) \nabla_{\nu} \nabla_{\sigma} \nabla_{\tau} K^{\tau}
$$

A Killing-Yano 3-form $Y_{\lambda_{1} \lambda_{2} \lambda_{3}}$ is defined as an antisymmetric tensor obeying

$$
\nabla_{\mu} Y_{\lambda_{1} \lambda_{2} \lambda_{3}}=\nabla_{[\mu} Y_{\left.\lambda_{1} \lambda_{2} \lambda_{3}\right]}
$$

From this equation we can show the following:

$$
\begin{gathered}
\nabla_{\mu} \nabla_{\nu} Y_{\lambda_{1} \lambda_{2} \lambda_{3}}=-2 R_{\mu \rho\left[\nu \lambda_{1}\right.} Y_{\left.\lambda_{2} \lambda_{3}\right]}^{\rho}, \\
0=R_{\mu \sigma_{1}[\nu}{ }^{\rho} Y_{\left.|\rho| \sigma_{2} \lambda\right]}+R_{\nu \sigma_{1}[\mu}{ }^{\rho} Y_{\left.|\rho| \sigma_{2} \lambda\right]}+\left(\sigma_{1} \leftrightarrow \sigma_{2}\right) .
\end{gathered}
$$

The following relation derived from the above is also useful:

$$
R_{\rho \sigma \lambda(\mu} Y_{\nu)}^{\rho \sigma}=2 R_{\rho(\mu} Y_{\nu) \lambda}^{\rho}
$$

[1] A. Sergyeyev and P. Krtous, Complete set of commuting symmetry operators for the Klein-Gordon equation in generalized higher-dimensional Kerr-NUT-(A)dS spacetimes, Phys. Rev. D 77, 044033 (2008).

[2] R. G. McLenaghan and P. Spindel, Quantum numbers for Dirac spinor fields on a curved space-time, Phys. Rev. D 20 , 409 (1979).

[3] I. M. Benn and P. Charlton, Dirac symmetry operators from conformal Killing-Yano tensors, Classical Quantum Gravity 14, 1037 (1997).

[4] I. M. Benn and J. Kress, First order Dirac symmetry operators, Classical Quantum Gravity 21, 427 (2004).

[5] M. Cariglia, P. Krtous, and D. Kubiznak, Commuting symmetry operators of the Dirac equation, Killing-Yano and Schouten-Nijenhuis brackets, Phys. Rev. D 84, 024004 (2011).

[6] P. Krtous, V.P. Frolov, and D. Kubiznak, Separation of Maxwell equations in Kerr-NUT-(A)dS spacetimes, Nucl. Phys. B934, 7 (2018).

[7] T. Houri, N. Tanahashi, and Y. Yasui, On symmetry operators for the Maxwell equation on the Kerr-NUT-(A)dS spacetime, Classical Quantum Gravity 37, 015011 (2020).
[8] L. Andersson, T. Bäckdahl, and P. Blue, Second order symmetry operators, Classical Quantum Gravity 31, 135015 (2014).

[9] Y. Michishita, On quantum numbers for RaritaSchwinger fields, Classical Quantum Gravity 36, 055010 (2019).

[10] S. Aksteiner and T. Bäckdahl, Symmetries of linearized gravity from adjoint operators, J. Math. Phys. (N.Y.) 60, 082501 (2019).

[11] B. Araneda, Symmetry operators and decoupled equations for linear fields on black hole spacetimes, Classical Quantum Gravity 34, 035002 (2017).

[12] B. Araneda, Generalized wave operators, weighted Killing fields, and perturbations of higher dimensional spacetimes, Classical Quantum Gravity 35, 075015 (2018).

[13] V. P. Frolov, P. Krtous, and D. Kubiznak, Black holes, hidden symmetries, and complete integrability, Living Rev. Relativity 20, 6 (2017).

[14] U. Semmelmann, Conformal Killing forms on Riemannian manifolds, arXiv:math/0206117. 